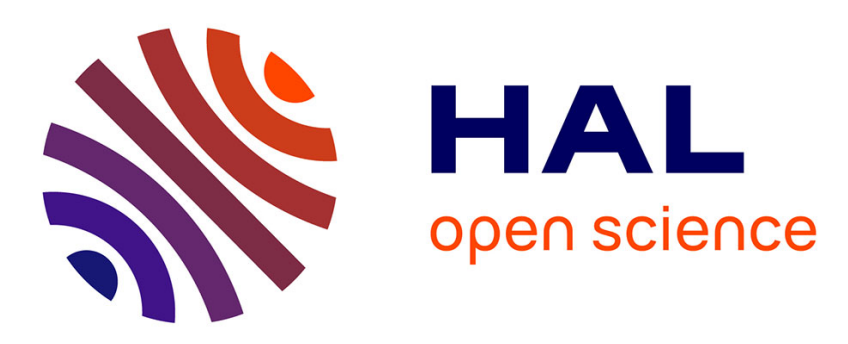

\title{
Impact of an Antifungal Insect Defensin on the Proteome of the Phytopathogenic Fungus Botrytis cinerea
}

Thomas Aumer, Sébastien N Voisin, Thomas Knobloch, Celine Landon, Philippe Bulet

\section{To cite this version:}

Thomas Aumer, Sébastien N Voisin, Thomas Knobloch, Celine Landon, Philippe Bulet. Impact of an Antifungal Insect Defensin on the Proteome of the Phytopathogenic Fungus Botrytis cinerea. Journal of Proteome Research, 2020, 19 (3), pp.1131-1146. 10.1021/acs.jproteome.9b00638 . hal-02994657

\section{HAL Id: hal-02994657 https://hal.science/hal-02994657}

Submitted on 18 Nov 2020

HAL is a multi-disciplinary open access archive for the deposit and dissemination of scientific research documents, whether they are published or not. The documents may come from teaching and research institutions in France or abroad, or from public or private research centers.
L'archive ouverte pluridisciplinaire HAL, est destinée au dépôt et à la diffusion de documents scientifiques de niveau recherche, publiés ou non, émanant des établissements d'enseignement et de recherche français ou étrangers, des laboratoires publics ou privés. 


\section{Impact of an antifungal insect defensin on the proteome of the}

2 phytopathogenic fungus Botrytis cinerea

3 Thomas Aumer', Sébastien N. Voisin ${ }^{2}$, Thomas Knobloch ${ }^{3}$, Céline Landon', Philippe Bulet ${ }^{12}$

4 ' CR Université Grenoble Alpes, Institute for Advanced Biosciences, Inserm U1209, CNRS

5 UMR 5309, Grenoble, France

6 '2 Plateforme BioPark d'Archamps, Archamps Technopole, Saint Julien en Genevois, France

$7 \quad{ }^{3}$ Bayer SAS, Bayer CropScience, Centre de Recherche de la Dargoire, Lyon, France

8 ' Centre de Biophysique Moléculaire, CNRS UPR 4301, Orléans, France

$10 *$ Corresponding Author

11 Dr Philippe Bulet

12 Plateforme BioPark d'Archamps, Bât. Le Forum 1, 260 Avenue Marie Curie, Archamps

13 Technopole, Saint Julien en Genevois Cedex, France

14 E-mail address: philippe.bulet@univ-grenoble-alpes.fr, philippe.bulet@ biopark-archamps.org 
ETD151, an analogue of the antifungal insect defensin heliomicin, is an antifungal peptide

17 active against yeasts and filamentous fungi. In order to decipher the mechanisms underlying its

18 molecular action on the phytopathogenic fungus Botrytis cinerea, a necrotrophic pathogen

19 responsible for gray mold disease, we investigated the changes in three-day old mycelia upon

20 treatment with different concentrations of ETD151. Optical and fluorescent microscopies were

21 used prior to establishing the peptide/protein profiles through two mass spectrometry

22 approaches: MALDI profiling, to generate molecular mass fingerprints as peptide signatures, and

23 a gel-free bottom-up proteomics approach. Our results show that a concentration of ETD151

24 above the half-maximal inhibitory concentration can alter the integrity of the mycelial structure

25 of $B$. cinerea. Furthermore, reproducible modifications of the peptide/protein composition were

26 demonstrated in the presence of ETD151 within a 1.5-16 kDa mass range. After the robustness

27 of LC-ESI-MS/MS analysis on B. cinerea mycelial extracts was confirmed, our analyses

28 highlighted 340 significantly modulated proteins upon treatment with ETD151 within a 4.8-

$29466 \mathrm{kDa}$ mass range. Finally, data mapping on KEGG pathways revealed the molecular impact

30 of ETD151 on at least six pathways. In particular, for the effect on oxidative phosphorylation, we

31 clearly demonstrated that ETD151 does not interact directly with the mitochondrial respiratory

32 chain.

34 Keywords: Insect defensin, antifungal defensin, Botrytis cinerea, proteomics, fungal infection, 35 heliomicin, mechanism of action 
Phytopathogenic fungi are a major concern for crops in agriculture. In the last decade,

39 phytopathology experts have alerted the scientific community to several species threatening food

40 security and biodiversity ${ }^{1}$. Every year, fungal pathogens destroy more than 125 million tons of

41 the world's top five major crops': rice (rice blast caused by Magnaporthe oryzae), wheat (rust

42 caused by Puccinia graminis), maize (smut caused by Ustilago maydis), potatoes (late blight

43 caused by Phytophthora infestans) and soybean (rust caused by Phakospora pachyrhizi). These

44 production losses could feed an additional 600 million people per year. Among the

45 phytopathogenic fungi, Botrytis cinerea (teleomorph Botryotinia fuckeliana) is a necrotrophic

46 pathogen responsible for gray mold disease causing significant crop losses in more than 200

47 plant species worldwide ${ }^{34}$. Ranked scientifically and economically as the second most important

48 fungal pathogen by the international phytopathological community ${ }^{5}$, B. cinerea generates

49 significant costs over a broad host range. Damage occurs in different stages of fruit and

50 vegetable production in open fields or in greenhouses and requires extensive disease control,

51 mainly by fungicide application. Concerns about this fungus have also increased due to the

52 recent discovery of $B$. cinerea fungicide-resistant strains in field populations ${ }^{6}$. The introduction

53 of site-specific fungicides almost 50 years ago revolutionized crop protection and has remained

54 until now the main approach for reducing fungal disease progression. However, plant pathogenic

55 fungi can adapt, leading to a tremendous loss of plant protection effectiveness. Fungicide

56 resistant pathogens have emerged over time and spread globally? Most important classes of

57 antifungals (e. g. benzimidazoles, strobilurins, hydroxyanilides) are concerned by the

58 development of resistance compromising the efficacy of crop protection. As a new major 
59 challenge, it is necessary to propose novel fungicides with innovative mechanisms of action or

60 alternative strategies with non-chemical productss

61 Scientists are investigating the field of antimicrobial peptides (AMPs), looking for new

62 molecules with novel targets, reduced toxicity and broad-spectrum activity for a low-rate use.

63 Also known as host defense peptides (HDPs), AMPs are key components of the innate immune

64 system of all organisms, protecting them against attacks by invading pathogens and stressors ${ }^{9}$.

65 Even if a wide range of AMPs have been identified, they can be globally classified according to

66 their amino acid composition, their structure and the organization of their disulfide bonds. One of

67 these classes, the CS $\alpha \beta$-defensins (Cysteine-stabilized $\alpha$-helix $\beta$-sheet), groups small, cationic,

68 cysteine-rich peptides with specific 3D organization and conserved disulfide bridge arrays ${ }^{10-12}$.

69 Intriguingly, the CS $\alpha \beta$-defensins have been found in plants and insects. Plant defensins are well-

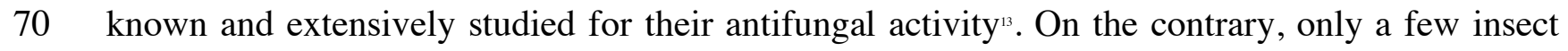

71 defensins have been reported to be exclusively antifungal, most of them exhibiting antimicrobial

72 activities (antibacterial and antifungal), with particular efficacy against Gram-positive bacteria ${ }^{14}$.

73 The first antifungal insect defensin to be described was drosomycin ${ }^{1516}$ from the fruit fly

74 Drosophila melanogaster (Diptera). It was followed by heliomicin ${ }^{17.8}$ from the tobacco budworm

75 Heliothis virescens (Lepidoptera), termicin ${ }^{190}$ from Pseudacanthotermes spiniger (Isoptera),

76 gallerimycin ${ }^{21}$ from the great wax moth larvae Galleria mellonella (Lepidoptera) and ARD1 ${ }^{22}$

77 from the larvae of Archaeoprepona demophon (Lepidoptera).

78 The promising in vitro antifungal activity of insect defensins against plant or human

79 pathogens, as well as their non-cytotoxicity, highlights their potential as candidates for crop

80 protection strategies ${ }^{23}$ and/or therapeutic applications ${ }^{24}$. In the 2000s, the French biotechnology

81 company EntoMed SA was the first to develop natural insect AMPs for the treatment of systemic 
82 fungal infections affecting immunocompromised patients $\mathrm{s}^{25}$. Heliomicin was the working model of

83 a cutting-edge optimization program based on phylogenic exploration and fine-tuned

84 mutagenesis. The results of their work, a 44 amino acid peptide, called ETD151, revealed

85 enhanced antifungal activity against Candida albicans and Aspergillus fumigatus as well as

86 additional activity against Cryptococcus neoformans, as compared to heliomicin ${ }^{21}$. ETD151 was

87 the principal product developed by EntoMed SA for treating antifungal infections ${ }^{26}$.

88 However, limited data has been gathered on the antifungal mechanism of action of insect

89 defensins, which include the inhibition of spore germination, the alteration of germ tube

90 elongation and the release of cytoplasmic content ${ }^{232}$. Comparisons based on structural analysis

91 and activity tests on fungal mutants, provided evidence of mechanical commonalities between

92 heliomicin, drosomycin and some antifungal plant defensins such as the radish defensin

93 RsAFP2 $2^{2 s-x}$. In contrast to insect defensins, the mechanisms of action of plant defensins have been

94 widely investigated. In most cases, the first step is the interaction with non-protein fungal

95 membrane partners such as sphingolipids or phospholipids ${ }^{3}$. Thereafter, plant defensins can

96 either be internalized or remain outside the fungal cells. Cell death can occur via multiple

97 mechanisms, induction of fungal membrane permeabilization, reactive oxygen species (ROS),

98 apoptosis, or mitochondrial functionality $\operatorname{loss}^{2}$. Effects differ depending on the target organism;

99 the mechanisms of action of the Medicago spp. defensin MtDef4 against the ascomycete fungi

100 Neurospora crassa and Fusarium graminearum ${ }^{33}$ are not the same. Upon exposure to antifungal

101 plant defensins, fungal cells will induce mechanisms to defend themselves against these adverse

102 effects. A family of conserved kinases, MAPK, regulate tolerance to plant defensins. In yeast,

103 the MAPK pathways are involved in filamentous growth, cell wall integrity (CWI) and the high

104 osmolarity/glycerol (HOG) response ${ }^{4}$. All of these elements provide a background for studying 
105 the mode of action of ETD151, an optimized peptide analogue of the two lepidopteran insect 106 antifungal peptides heliomicin and ARD1.

107 To decipher the mechanism of action of ETD151 on the fungal phytopathogen B. cinerea, a 108 proteomics approach based on matrix-assisted laser desorption/ionization mass spectrometry 109 (MALDI-MS) and liquid chromatography-mass spectrometry coupled to electrospray ionization 110 (ESI-LC-MS) was used. The elucidation of the $B$. cinerea genome was a significant 111 breakthrough for the proteomics research community and has provided for the accurate 112 identification of proteins in this fungus, mainly from mycelial samples ${ }^{35-38}$. In the past ten years, 113 several methodological developments and technological advances in Botrytis proteomics have 114 offered effective new tools for achieving a better understanding of $B$. cinerea biology and how 115 its proteins are involved in pathogenic biological processes ${ }^{39,40}$. Most of this work focused on the 116 secretome or virulence factors produced by the fungus, like cell wall degrading enzymes, 117 considered as key elements in the different steps of the infection cycle of phytopathogenic 118 fungi ${ }^{41,42}$.

119 Due to the dynamic nature of the proteome, the addition of an antifungal compound should 120 affect processes occurring at the surface and / or within the fungal cell ${ }^{43}$. In this work we compare 121 the protein profiles of extracts of fresh mycelia treated with ETD151 vs untreated cultures. This 122 proteomics study gives insight into the mechanism of action of this insect defensin on the fungus 123 B. cinerea. 


\section{MATERIALS AND METHODS}

\section{Chemical reagents}

127 A liquid minimum medium based on Tanaka's medium $\mathrm{B}^{4+45}$ and a Potato Dextrose Broth 128 medium diluted 1:4 in water (25\% PDB) were prepared using reagents from Sigma-Aldrich 129 (St. Louis, MO, USA). Potato Dextrose Agar (PDA) was purchased from Oxoid (Basingstoke, $130 \mathrm{UK})$. The reagents $\alpha$-cyano-4-hydroxycinnamic acid (4-HCCA), ammonium bicarbonate (ABC), 131 dithiothreitol (DTT), iodoacetamide (IAA), HPLC-grade dimethyl sulfoxide (DMSO) and LC132 MS-grade formic acid (FA) were from Sigma-Aldrich. SYTOX Green Nucleic Acid Stain (5 mM 133 in DMSO) was from Thermo Fisher Scientific (Waltham, MA, USA), RapiGest SF surfactant 134 was from Waters (Milford, MA, USA) and sequencing-grade modified trypsin was from 135 Promega (Madison, WI, USA). Acetonitrile (ACN), ethanol absolute anhydrous (EtOH), 136 trifluoroacetic acid (TFA), all LC-MS grade quality or higher, were obtained from Carlo Erba

137 Reagents (Val de Reuil, France). Protein Calibration Standard I (ProtMix) and Peptide 138 Calibration Standard II (PepMix) were purchased from Bruker Daltonics (Germany). For all 139 experiments, MilliQ water was used (Billerica, MA, USA).

\section{$141 \quad$ Biological material}

142 Spores of Botrytis cinerea isolated from the field and cryopreserved in 10\% DMSO were 143 provided by the La Dargoire Research Center (Bayer CropScience, France). All spores of 144 B. cinerea used in this study were derived from the same initial batch. For each experiment, a 145 cryotube was thawed and the stock solution of spores was diluted with sterile MilliQ water 146 resulting in a final concentration of $1.5 \times 10^{5}$ spores per $\mathrm{mL}(\mathrm{sp} / \mathrm{mL})$ in the presence of $0.1 \%$ 147 DMSO. In order to have fresh fungal material, Falcon ${ }^{\circledR}$ culture petri dishes (Corning, NY, USA) 
148 containing PDA solid medium were inoculated with $150 \mu \mathrm{L}$ of the appropriate spore suspension.

149 Culture dishes were left in the dark for seven days in an incubator (Memmert, Schwabach,

150 Germany) maintained at $20^{\circ} \mathrm{C}$ and at $70 \%$ level of hygrometry. These parameters were defined

151 as the culture conditions for all our experiments. To prepare a fresh spore solution, spores were

152 harvested from one seven-day old culture dish using a Falcon ${ }^{\circledR}$ cell scraper (Corning), filtered on

153 a Falcon ${ }^{\circledR}$ nylon $100 \mu \mathrm{m}$ cell strainer (Corning), counted twice with a Malassez chamber and 154 adjusted to a final concentration of $500 \mathrm{sp} / \mathrm{mL}$ in minimal medium.

\section{Antifungal assay}

157 The bioactive peptide ETD151, provided by Dr. Philippe Bulet, was produced by high cell 158 density fermentation of a modified Saccharomyces cerevisiae strain developed by the former 159 company EntoMed S.A. (Illkirch, France) and preserved as a stock solution in saline buffer. ETD

160151 was desalted by solid phase extraction using Sep-Pak $\mathrm{C}_{18}$ Classic Cartridges (Waters)

161 following the manufacturer's instructions. Solutions from 250 to $0.49 \mu \mathrm{M}$ of ETD151 were 162 prepared in sterile MilliQ water. Antifungal assays on $B$. cinerea were performed in sterile 96163 well plates (TPP Techno Plastic Products AG, Trasadingen, Switzerland). To assess inhibition of 164 mycelium growth, microplate wells were filled with $135 \mu \mathrm{L}$ of the fresh suspension at $500 \mathrm{sp} / \mathrm{mL}$ 165 prior to incubation in the conditions reported in the Biological material section. Addition of 15 $166 \mu \mathrm{L}$ of the ETD151 solutions, giving final concentrations of 25 to $0.049 \mu \mathrm{M}$, occurred three days 167 later. The plate was gently shaken and incubated for four additional days. Evolution of the assay 168 was monitored daily by absorbance measurement at $630 \mathrm{~nm}$ with an ELx808 microplate reader 169 (BioTek, Winooski, VT, USA). The dose-response curve was generated with Microsoft Excel 
170 software and XLfit plug-in (release 5.4). Data were adjusted according to the "Dose-response

171 one site -205 " model to determine the half-maximal inhibitory concentration $\left(\mathrm{IC}_{\mathrm{s} 0}\right)$.

172

$173 \quad$ Fluorescence microscopy

174 A fresh spore solution at $1 \times 10^{4} \mathrm{sp} / \mathrm{mL}$ was prepared in a $25 \%$ PDB medium as reported in the

175 Biological material section and $200 \mu \mathrm{L}$ were distributed in several microplate wells. Spores were 176 supplemented with SYTOX Green (final concentration of $30 \mu \mathrm{M}$ ) prior to shaking and 177 incubation for 24 hours. Young mycelia of $B$. cinerea were treated by addition of ETD151 at 178 final concentrations of 0.78 and $3.12 \mu \mathrm{M}$ before a new incubation period of 24 hours. 179 Morphological changes were monitored in transmitted light (TransLight50, 30 ms exposure) with 180 an ImageXpress Micro XL fully automated inverted microscope (Molecular Devices, San Jose, 181 CA, USA) equipped with a Plan Fluor ELWD 40x objective (Nikon). SYTOX Green 182 fluorescence was observed with a Green Fluorescent Protein filter (excitation wavelength, $183472 \mathrm{~nm}$; emission wavelength, $520 \mathrm{~nm}$; $100 \mathrm{~ms}$ exposure).

\section{Sample preparation for proteomic studies}

186 In a 96-well plate (TPP product), $135 \mu \mathrm{L}$ of the fresh suspension at $500 \mathrm{sp} / \mathrm{mL}$ in minimum 187 medium was distributed in six rows of 10 wells each. Mycelia were cultivated by maintaining the 188 plate for three days in the growth conditions defined previously. Subsequently, $15 \mu \mathrm{L}$ of sterile 189 MilliQ water was added to the first row as an untreated control. The five other rows were treated 190 with five different concentrations of ETD151, ranging from $31.2 \mu \mathrm{M}$ to $1.95 \mu \mathrm{M}$. The 191 experimental plate was maintained for 24 hours in the previously described growth conditions 
192 until protein extraction. Each microplate was monitored daily by measuring the absorbance at

$193630 \mathrm{~nm}$ with the ELx808 microplate reader (BioTek).

194 Protein extraction

195 For each plate, the fungal material (mycelium) from each of the 60 wells was collected by 196 manual pipetting. For each experimental condition (untreated or treated with different 197 concentrations of ETD151), 10 individual wells of a same row were pooled into a single $1.5 \mathrm{~mL}$ 198 Eppendorf tube and maintained on ice until use. The fungal material was pelleted by 199 centrifugation for $4 \mathrm{~min}$ at $13,000 \mathrm{rpm}$ and $4^{\circ} \mathrm{C}$. The fungal pellet was washed twice by up-and200 down pipetting with $1 \mathrm{~mL}$ of sterile MilliQ water before adding $1 \mathrm{~mL}$ of $70 \% \mathrm{EtOH}$. The washed 201 mycelia were stored overnight at $-80^{\circ} \mathrm{C}$. The fungal material was disrupted using a Mill-Mix 20

202 system (Domel, Železniki, Slovenia). The mycelia were ground in 2-mL tubes filled with $0.5 \mathrm{~mm}$ 203 zirconium oxide beads for $10 \mathrm{~min}$ at $30 \mathrm{~Hz}$. After centrifugation for $4 \mathrm{~min}$ at 13,000 rpm and $2044^{\circ} \mathrm{C}$, the pellets were collected and dried under CentriVap vaccum (Labconco, Kansas City, MO, 205 USA). Finally, the proteins were extracted by incubating the dried fungal pellet in the presence 206 of $2 \mathrm{M}$ acetic acid, prepared in $50 \% \mathrm{ACN}$, for $4 \mathrm{~h}$ at $4^{\circ} \mathrm{C}$ under gentle shaking. After incubation, 207 the extracts were centrifuged for $4 \mathrm{~min}$ at $13,000 \mathrm{rpm}$ and $4^{\circ} \mathrm{C}$ and the protein supernatants were 208 stored at $4^{\circ} \mathrm{C}$ until use.

210 Sample preparation for MALDI mass spectrometry analyses

211 For MALDI MS analyses, a fresh solution of matrix was prepared by adding, up to saturation, 212 a few mg of 4-HCCA in $1 \mathrm{~mL}$ of Biotyper solution (Bruker Daltonics) composed of 50\% ACN 213 and $2.5 \%$ TFA. The samples to be analyzed $(0.5 \mu \mathrm{L})$ were spotted and immediately covered with $2140.5 \mu \mathrm{L}$ of the matrix solution on an MTP 96 polished steel MALDI target (Bruker Daltonics). 
215 Calibration of the instrument was performed using PepMix complemented with ProtMix. The 216 sample spots were quickly dried under mild vacuum prior to analysis.

218 Peptide mass fingerprint of $B$. cinerea mycelium by MALDI-TOF mass spectrometry

219 Mass spectra from protein supernatants described in the Protein extraction section were 220 acquired on an AutoFlex III Smartbeam instrument (Bruker Daltonics) using the FlexControl 3.4 221 software (Bruker Daltonics) in positive linear mode. The instrument was set up with the 222 following parameters: $200 \mathrm{~Hz}$ laser at a $40 \%$ global attenuation offset, $20 \mathrm{kV}$ source voltage 1, $22318.5 \mathrm{kV}$ source voltage $2,9 \mathrm{kV}$ lens voltage, $1.82 \mathrm{kV}$ linear detector voltage, $140 \mathrm{~ns}$ of pulsed 224 ion extraction delay and 600 Da detector gating. MALDI MS spectra were recorded at the mass 225 range $600 \mathrm{Da}$ to $18 \mathrm{kDa}$ by summing two sets of 1,000 laser shots. Data were previewed using 226 the FlexAnalysis 3.4 software (Bruker Daltonics) and reprocessed in mMass software ${ }^{\star 6}$ (version

227 5.5.0) Average spectra were generated from three selected biological replicates (from a pool of 228 six), based on their fingerprint similarities and their intensities (maximum arbitrary intensity $>$ $22910,000)$. The average spectra were cropped from 1,500 to $16,000 \mathrm{~m} / \mathrm{z}$, baseline correction was 230 performed with a relative offset of 100 and a precision of 40, along with a smoothing according 231 to the Savitzky-Golay algorithm (window size $5.0 \mathrm{~m} / \mathrm{z}$ in one cycle). The peaks were 232 automatically picked using a signal-to-noise $(\mathrm{S} / \mathrm{N})$ ratio of 2.0 , a picking height of 90 and an 233 application of baseline. Finally, the average mass spectra were concomitantly and manually 234 curated to select 100 representative ions. 
Proteins were digested using the modified procedure reported by Masson and colleagues ${ }^{47}$.

238 Briefly, the protein supernatants were dried under vacuum (Labconco), and then suspended with

239 gentle shaking in an ammonium bicarbonate (ABC) buffer (50 mM, pH 7.5) supplemented with

$240 \quad 0.1 \%$ RapiGest surfactant. The proteins were reduced with $280 \mathrm{mM}$ dithiothreitol in ABC buffer

241 for $30 \mathrm{~min}$ at $56^{\circ} \mathrm{C}$ in the dark prior to alkylation with $480 \mathrm{mM}$ iodoacetamide in $\mathrm{ABC}$ buffer for

$24230 \mathrm{~min}$ at room temperature in the dark. Digestions were carried out for $1 \mathrm{~h}$ at $37^{\circ} \mathrm{C}$ by adding

$2430.5 \mu \mathrm{g}$ of trypsin $(0.1 \mu \mathrm{g} / \mu \mathrm{L}$ in $\mathrm{ABC}$ buffer $)$. Following this initial digestion, $0.5 \mu \mathrm{g}$ of trypsin

244 was added before overnight incubation at $37^{\circ} \mathrm{C}$. To stop proteolysis and cleave the RapiGest

245 surfactant, samples were acidified with $8 \mu \mathrm{L}$ of a $10 \%$ TFA and $20 \%$ ACN solution and

246 incubated for $30 \mathrm{~min}$ at $37^{\circ} \mathrm{C}$. Finally, samples were centrifuged for $10 \mathrm{~min}$ at $13,000 \mathrm{rpm}$ at

247 room temperature. The digests were transferred into injection vials (Agilent Technology, France)

248 containing $5 \mu \mathrm{L}$ of $2 \%$ ACN acidified with a solution of $0.1 \%$ TFA.

250 Proteomic analyses by nano-LC-ESI-MS/MS

251 Nano-LC-ESI-MS/MS instrumentation, software, chromatographic columns and other items 252 were obtained from Thermo Fisher Scientific. Peptides were analyzed using an Ultimate 3000 253 nanoflow HPLC system coupled to a Q-Exactive Orbitrap high-resolution mass spectrometer, 254 controlled by Xcalibur 2.2, Chromeleon Xpress 6.80 and Thermo QExactive Tune 2.5 software. 255 Nano-LC separations were performed according to Pisani and colleagues ${ }^{48}$. Briefly, the digests 256 were loaded at a flow rate of $10 \mu \mathrm{L} / \mathrm{min}$ for 6 min onto a PepMap $100 \mathrm{C}_{18}$ precolumn $(300 \mu \mathrm{m} \mathrm{x}$ $2575 \mathrm{~mm}, 5 \mu \mathrm{m})$ and separated at a flow rate of $300 \mathrm{~nL} / \mathrm{min}$ onto an Acclaim PepMap $100 \mathrm{C}_{18}$ 258 nanoviper column $(75 \mu \mathrm{m} \times 250 \mathrm{~mm}, 3 \mu \mathrm{m})$ maintained at $35^{\circ} \mathrm{C}$. Peptides were eluted using a 259 multi-step linear gradient from $2 \%$ to $32 \%$ and from $32 \%$ to $65 \% \mathrm{ACN}$ in $0.1 \%(\mathrm{v} / \mathrm{v}$ ) formic acid 
260 for $94 \mathrm{~min}$ and $5 \mathrm{~min}$, respectively. For MS analyses, the mass spectrometer was operating in

261 positive mode and data-dependent acquisition. The instrument acquired a full-range MS scan

262 from 380 to $2000 \mathrm{~m} / \mathrm{z}$ (70,000 resolution, AGC target 3.10', maximum IT $200 \mathrm{~ms})$ and then

263 fragmented the top ten peptide ions in each cycle (17,500 resolution, AGC target 2.10,

264 maximum IT $100 \mathrm{~ms}$, intensity threshold 4.10, excluding charge-unassigned ions, Normalized

265 Collision Energy 27). Parent ions were then excluded from MS/MS for the next $15 \mathrm{~s}$.

266

267 Databases searching and annotation

268 All MS and MS/MS spectral data were analyzed using Proteome Discoverer software 269 (version 2.2.0.388, Thermo Fisher Scientific). Processing and consensus workflows were 270 adapted from manufacturer's basic settings. The acquired MS/MS spectra were matched using

271 the SEQUEST $\mathrm{HT}^{*}$ search algorithm (University of Washington) against the EnsemblFungi ${ }^{\text {io }}$ 272 Botrytis cinerea B05.10 protein sequence database (http://fungi.ensembl.org/Botrytis cinerea).

273 The release 42 downloaded on February 16, 2019 contains 13,749 entries. The spectrum

274 identification considered the following parameters: trypsin as proteolytic enzyme with a 275 maximum of two missed cleavages, 6 and 144 amino acids as minimum and maximum peptide 276 lengths, and a tolerance of $10 \mathrm{ppm} / 0.02 \mathrm{Da}$ for precursor and fragment masses, respectively. 277 Cysteine carbamidomethylation was defined as a static modification. Methionine/tryptophan 278 oxidation, serine/threonine/tyrosine phosphorylation and C-terminal amidation were defined as 279 dynamic modifications. False discovery rates (FDRs), referred to as q-values, and posterior error 280 probabilities (PEPs) for peptide spectral matches (PSMs) were calculated and filtered in the 281 Percolator node using a decoy database ${ }^{5152}$. Peptides and identified proteins were respectively 282 validated based on high-confidence (FDR $<1 \%$ ) and medium-confidence criteria (FDR $<5 \%$ ). 
283 Protein grouping was applied to regroup protein candidates covered by the same set or subset of 284 identified peptides under a representative 'master' protein. Annotations were implemented

285 through Proteome Discoverer using Gene Ontology (biological process, molecular function, 286 cellular compartment), protein families from Pfam and biochemical pathway maps from the 287 Kyoto Encyclopedia of Genes and Genomes (KEGG).

289 Protein quantification and functional analysis

290 Label-free quantification was performed using the Proteome Discoverer software. 291 Chromatographic alignment for LC/MS mapping features, created by the Minora Feature 292 Detector for each individual file, was achieved with a maximum retention time shift of 10 min 293 and a mass tolerance of $10 \mathrm{ppm}$. Protein abundance was determined on intensity of precursor 294 ions of unique and razor peptides.

295

296 Activity of complex I/ III and II/III of the respiratory transport chain

297 Mitochondrial preparation

298 Two liters of fresh spores of $B$. cinerea were cultured in glucose yeast mycological peptone 299 medium and incubated in Erlenmeyer flasks with stirring for $72 \mathrm{~h}$ at $22^{\circ} \mathrm{C}$. Mycelia were filtered 300 through a $200 \mu \mathrm{m}$ nylon cloth NITEX (Sefar AG, Heiden, Switzerland), before washing with 301 MilliQ ice water, spinning and weighing. The following steps were performed in a cold room $302\left(4^{\circ} \mathrm{C}\right)$ to preserve functionality of mitochondria. Mycelia were resuspended in a $0.5 \mathrm{M}$ 303 sucrose/1 mM EDTA/0.3\% BSA/20 mM MOPS wash buffer adjusted to $\mathrm{pH}$ 7.4. After addition 304 of $5 \mathrm{mM}$ cysteine, the mixture was ground with $1 \mathrm{~mm}$ glass beads for three cycles of $30 \mathrm{~s}$ with 3051 min pauses to cool down the suspension. Fungal material was filtered on a $100 \mu \mathrm{m}$ nylon cloth 
306 (Sefar) and beads were rinsed with the washing buffer, prior to centrifugation of the filtrate for

$30710 \mathrm{~min}$ at $1,500 \mathrm{~g}$. The supernatant was filtered on a $150 \mu \mathrm{m}$ nylon cloth (Sefar) prior to

308 centrifugation for $20 \mathrm{~min}$ at $15,000 \mathrm{~g}$. The mitochondrial pellet was resuspended in the wash

309 buffer and fractionated in 2-mL aliquots prior to purification on a $1.8 \mathrm{M} / 1.2 \mathrm{M}$ sucrose gradient,

310 involving centrifugation for $45 \mathrm{~min}$ at $36,000 \mathrm{~g}$ followed by slow deceleration. Purified

311 mitochondrial fractions at the sucrose bilayer interface were diluted in the wash buffer and

312 centrifuged for $15 \mathrm{~min}$ at $36,000 \mathrm{~g}$. Pellets were combined prior to a new washing step to obtain

313 a single purified fraction of mitochondria.

314 Measurement of inhibition of complex I/III and II/III by colorimetry

315 The activity of a molecule as a possible electron transport chain complex inhibitor was

316 measured by a potassium ferricyanide-based colorimetric test ${ }^{\mathrm{t} 3}$ in the presence of $\alpha$-ketoglutarate

317 and succinate, substrates of complexes I and II, respectively. Viability of fresh mitochondria was

318 evaluated in 96-well microplates (Greiner Bio One). For complex I, $3 \mu \mathrm{L}$ of the purified fraction

319 was diluted in $400 \mu \mathrm{L}$ of Mix I solution (Supplementary data S1) before depositing $88 \mu \mathrm{L}$ of this

320 mixture into 4 control wells and adding the $0.05 \mathrm{M} \alpha$-ketoglutarate substrate. After a 3-min

321 incubation at room temperature, the absorbance was measured for 5 min at $405 \mathrm{~nm}$ (SpectraMax

322 Plus 384, Molecular Devices). Data was converted to a slope using a linear regression model.

323 Complex II activity was evaluated in the same way using Mix II (Supplementary data S1) and

324 the appropriate substrate. For this assay, the ETD151 peptide diluted in sterile Milli-Q water was

325 compared to two standard inhibitors diluted in DMSO: antimycin A and boscalid. From a

326 dilution range (factor of 3.5) prepared upstream, $2 \mu \mathrm{L}$ of the molecule to test (50X) was

327 deposited in a well containing $88 \mu \mathrm{L}$ of fresh mitochondria in Mix I or Mix II, for complex I or

328 complex II, respectively. Immediately for complex I or after $7 \mathrm{~min}$ of incubation for complex II, 
$32910 \mu \mathrm{L}$ of $0.05 \mathrm{M}$ substrate were added before incubation for $3 \mathrm{~min}$ at room temperature. After a 330 brief stirring, the mitochondrial activity was measured as previously described. 


\section{Fungal growth inhibition and morphological changes in Botrytis cinerea}

334 Several antifungal defensins from insects, like heliomicin ${ }^{23}$ and drosomycin ${ }^{15}$, or from plants, 335 such as RsAFP2 ${ }^{s 4}$ and VvAMP2 ${ }^{\text {ss }}$, have been reported to be active against Botrytis cinerea at a 336 micromolar range. We have previously shown that ETD151 exhibits a broad-spectrum of 337 activities against several yeasts and filamentous fungi ${ }^{22}$ at the micromolar range. However, 338 ETD151 had not been tested on $B$. cinerea. In this study, the antifungal activity of ETD151 was 339 assessed in vitro against three-day-old mycelia of $B$. cinerea prepared from a fresh spore 340 solution. In our experimental conditions, growth inhibition reaches a maximum, $78 \%$ as 341 measured by optical density, at $6.25 \mu \mathrm{M}$ of ETD151 (Figure 1A). Based on this value, the half342 maximal inhibitory concentration $\left(\mathrm{IC}_{\mathrm{s0}}\right)$ was estimated to be $0.59 \mu \mathrm{M}$.

343 The antifungal action of ETD151was monitored by optical microscopy (Figure 1B). At 0.78 344 and $3.12 \mu \mathrm{M}$, ETD151 is able to promote lysis and the release of intracellular content at some 345 points of the growing mycelium, preferentially at the apical end of the hyphae (Fig. 1B, b-c).

346 Such a leakage of cytosolic material suggests a loss of the cell wall integrity. An equivalent 347 observation has already been reported for heliomicin and drosomycin on B. cinerea spores, but 348 for these molecules, comparatively weaker effectiveness against the mycelium was recorded ${ }^{23}$. 349 Thus, our data confirm the enhanced activity of ETD151 compared to heliomicin, the reference 350 molecule.

351 The permeabilization of the fungal membrane has emerged as a trend in the antifungal mode of 352 action of plant defensins ${ }^{3256}$. The results obtained on $B$. cinerea mycelia treated with the insect 353 defensin homologue ETD151 suggest a similar mode of action. Nevertheless, it remains unclear 354 if ETD151 is internalized or if it remains outside the fungal cells. To assess whether ETD151 is 
355 able to disturb membrane integrity/permeability, the SYTOX Green nucleic acid stain, a cell-

356 impermeant fluorophore, was used. Compared to the control experiment (Fig. 1B, g), in the

357 presence of ETD151, the dye penetrated locally the hyphae and emitted a green-fluorescent

358 signal revealing a compromised plasma membrane (Fig. 1B, h-i).

359 In addition, ETD151 reduced hyphal elongation with a concomitant disorder in hyphal

360 branching at both tested concentrations (Fig. 1B, e and f, respectively 0.78 and $3.12 \mu \mathrm{M}$ ).

361 Development of the $B$. cinerea mycelium post-treatment highlights the fungistatic aspect of

362 ETD151 activity and may explain the inhibition plateau observed with the fungal growth curve

363 (Fig. 1A). Swelling of hyphae was also noticed with a particularly strong phenotype at 3.12 $\mu \mathrm{M}$

364 (Fig. 1B, f). These morphological changes defined ETD151 as a morphogenic defensin of

365 B. cinerea. This is homologous to observations reported for several plant defensins (RsAFP2,

366 HsAFP1, MsDef1) in filamentous fungi ${ }^{57-59}$ and confirms commonalities with the antifungal action

367 of the insect defensins. Results obtained after this first series of analyses showed that there are

368 perturbations of the mycelium physiology and morphology, as the optical microscopy pictures

369 and the uptake of the SYTOX Green nucleic acid stain differed between all treatment conditions.

370 Thevissen and colleagues ${ }^{28}$ demonstrated through an ELISA-based assay with Pichia pastoris

371 that heliomicin and RsAFP2 interact with fungal membrane lipids called glucosylceramides. All

372 of our structural and biological data support a common mechanism of action for these three

373 defensins, and lead us to suggest that ETD151 interacts with the same fungal partners.

374 To study the antifungal effects of ETD151 on the intracellular protein content, we used a

375 proteomics study based on a two-step MS workflow, and conducted on B. cinerea mycelial

376 extracts. Firstly, a comparison of mass spectrometry (MS) molecular mass fingerprints was

377 conducted by MALDI-MS. This first step was designed to follow the evolution of the peptide 
378 protein profiles within the mass range of $600 \mathrm{Da}$ to $18 \mathrm{kDa}$. Secondly, a bottom-up proteomics 379 analysis by nano-LC-ESI-MS/MS was performed to identify and characterize the proteins 380 changes. The concentration range from 0.19 to $3.12 \mu \mathrm{M}$, which includes the $\mathrm{IC}_{\mathrm{s} 0}$ and covers the 381 entire inhibition profile of ETD151, was adopted for a dose-response approach. 
A

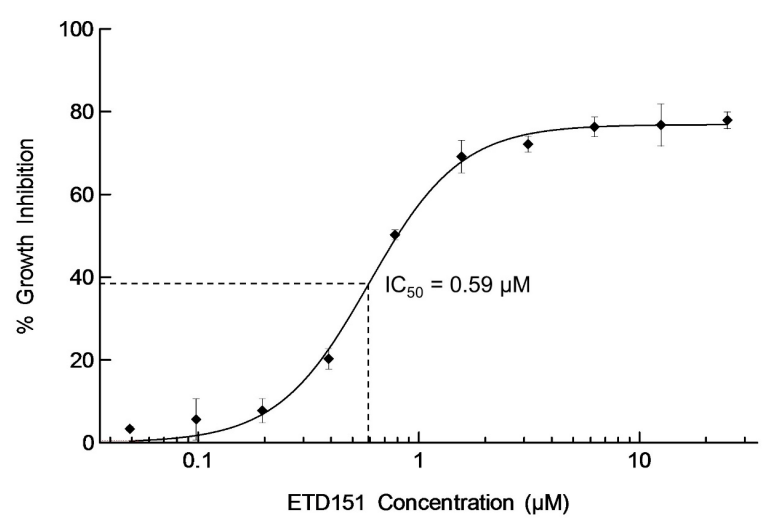

382

383

384

385

386

390 hour $(\mathrm{a}, \mathrm{b}, \mathrm{c})$ and $24 \mathrm{~h}(\mathrm{~d}, \mathrm{e}, \mathrm{f})$ of incubation in Potato Dextrose Broth diluted by 1/4 ${ }^{\text {th }}$. Mycelia 391 were incubated in the absence (control experiment, a, d) or the presence of ETD151 at a final 392 concentration of $0.78 \mu \mathrm{M}(\mathrm{b}, \mathrm{e})$ and $3.12 \mu \mathrm{M}(\mathrm{c}, \mathrm{f})$. SYTOX Green signal was measured by 393 fluorescence microscopy $24 \mathrm{~h}$ post-treatment $(\mathrm{h}, \mathrm{i})$ on the same experimental plate wells as the 394 control experiment $(\mathrm{g})$. Arrows indicate lysis of hyphae. Scale bar $=50 \mu \mathrm{m}$.
B

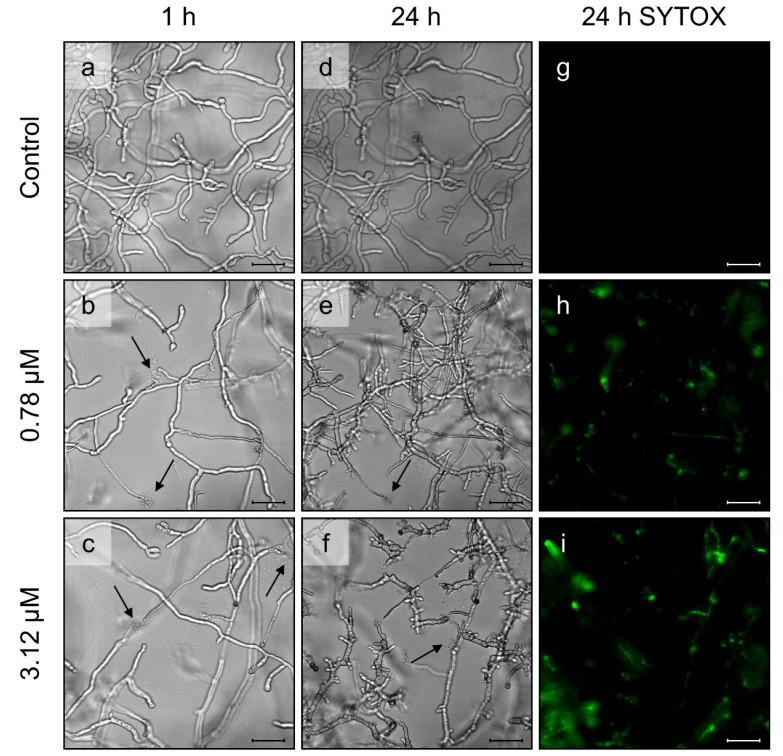

Figure 1: Growth inhibition and morphogenic alterations induced by ETD151 in B. cinerea

(A) Growth inhibition (\%) was evaluated on B. cinerea three-day-old mycelia produced from fresh spores incubated in modified Tanaka. The dose-response curve was determined by incubating mycelia with ETD151 concentrations ranging from 0.049 to $25 \mu \mathrm{M}$ (final concentration in $\log _{10}$ ) for four days. The dotted lines indicate the $\mathrm{IC}_{50}$. Data are the means of triplicate measurements. Error bars denote standard deviation. (B) Morphogenic alterations of $B$. cinerea mycelia obtained from a fresh culture of spores were observed microscopically after one 


\section{Protocol development to prepare $B$. cinerea mycelial extracts}

396 Fungal culture, extraction and MALDI-MS analysis protocols have been developed for this

397 study. The extraction protocol is inspired by the work of Schulthess and colleagues ${ }^{60}$, using $35 \%$

398 formic acid for the BioTyping of clinical molds. The objective was to establish the optimal

399 parameters for recording the most representative MALDI peptide signatures/molecular mass

400 fingerprints (MFPs) of $B$. cinerea, with or without different concentrations of ETD151. The

401 following parameters were reviewed: the starting concentration of $B$. cinerea spores, the mycelial

402 culture duration in microplate, the treatment duration with ETD151 and the extraction process to

403 generate mycelial extracts. The development of the protocols was focused on the conditions

404 providing the most reproductible MFPs and with a rich molecular diversity. We settled on a

405 starting concentration of $500 \mathrm{sp} / \mathrm{mL}$ prior to three days of growth in microplate and a 24h-

406 treatment of the mycelia with ETD151. Peptides/proteins were extracted by grounding the

407 mycelia with zirconium oxide beads, drying the pellet and incubating it in the presence of $2 \mathrm{M}$

408 acetic acid solution prepared in 50\% ACN (personal communication, Victor Masson and

409 Philippe Bulet). The different parameters for MALDI-MS analysis were optimized for an

410 optimal detection (in terms of sensitivity, resolution, reproducibility and accuracy) of molecular

411 ions between $1,500-12,000$ mass $\mathrm{m} / \mathrm{z}$ of the $B$. cinerea mycelial extracts.

412

$413 \quad$ Peptide mass fingerprint of $B$. cinerea mycelium in response to ETD151

414 Once the experimental methodology had been established, MALDI-MS analyses were 415 performed directly on $B$. cinerea mycelial peptide/protein extracts, and restricted to peptides and 416 small proteins $(<20 \mathrm{kDa})$ as a preliminary approach to assess the antifungal effect of ETD151 at 417 the molecular scale. Three-day-old mycelia were incubated for $24 \mathrm{~h}$ with the defensin following 
418 the previous concentration range, from 0.19 to $3.12 \mu \mathrm{M}$ final concentrations. Average spectra $419(n=3)$ were generated from biological replicates to first, highlight modifications of the 420 peptide/protein composition in the presence of ETD151 and second, visualize a putative dose421 response effect (Figure 2). For each concentration, a manual selection from automatic peak 422 labeling resulted in a representative peak list of 100 ions. Similarities between MALDI MFPs 423 were calculated following the conservation of representative ions (Supplementary data S2).

424 In the presence of $0.19 \mu \mathrm{M}$ of ETD151, no strict molecular change was observed on the MFPs. 425 Compared to the untreated mycelia, MFPs were very similar with $91 \%$ identity (Fig. 2A). This 426 observation is consistent with the very reduced growth inhibition effect (less than 10\%) observed 427 at this dose (Fig. 1A). From $0.39 \mu \mathrm{M}$ of ETD151, the antifungal activity of ETD151 has a 428 significant impact on the MFPs recorded. Several modifications were highlighted on the MFPs 429 compared to untreated mycelia, with identity dropping to $68 \%$ (Fig. 2B). Differences in the 430 peptide/protein profiles are significant in particular within the 6,000-9,000-mass range 431 (Supplementary data S3). At $0.39 \mu \mathrm{M}$, two sets of peptides/proteins within the 9,000-10,000 and 432 14,000-17,000 mass ranges emerged; those were not detectable in the control MFP or at an 433 ETD151 concentration of $0.19 \mu \mathrm{M}$.

434 At $0.39 \mu \mathrm{M}$ or higher doses of ETD151, the MALDI-MS spectra showed similar overall MFPs 435 (Fig. 2C). Indeed, the dose of $0.39 \mu \mathrm{M}$ shares $92 \%, 84 \%$ and $77 \%$ identity with the $0.78,1.56$ 436 and $3.12 \mu \mathrm{M}$ doses, respectively. Several ions within the 9,000-10,000 and 14,000-17,000 mass 437 ranges display intensity variation according to the concentration of the defensin with intensities 438 increasing as the concentration reaches $0.39 \mu \mathrm{M}$ (Supplementary data S2). These results suggest 439 that MALDI-MS could be a rapid and simple method to assess the impact of an antifungal 440 molecule on its fungal target. The modifications of the MFPs observed between the proteins 
441 extracted from the control experiment versus $B$. cinerea mycelia treated with different doses of 442 ETD151 also revealed that ETD151 modified the peptide/protein profiles of $B$. cinerea mycelia, 443 in a dose dependent manner up to a concentration that is more than the $\mathrm{IC}_{s 0}(0.59 \mu \mathrm{M})$.

444 Despite the extraction method used, including removal of the culture supernatant and two 445 successive washing steps of the mycelia, the ion observed at $\mathrm{m} / \mathrm{z} 4,839$ corresponds to ETD151. 446 It is worth mentioning that the intensity of this ion does not decrease with two additional washes 447 (unpublished data). This suggests that in our experimental conditions, ETD151 was interacting 448 with the membrane of the altered mycelium of B. cinerea, or was entering the mycelium. Note 449 that the arbitrary intensity (a.i.) of the ETD151 ion increases with the concentration of ETD151 450 used, from $1.9 \cdot 10^{3}$ a.i. at $0.19 \mu \mathrm{M}$ to 174.103 a.i. at $3.12 \mu \mathrm{M}$ (Supplementary data S2).

451 As important modifications of the MFPs are observed within the highest molecular mass range 452 detectable in our MALDI-MS conditions, this preliminary approach attests to the antifungal 453 effect of ETD151 on B. cinerea and prompted us to analyze the mycelial extract of B. cinerea 454 treated with ETD151 at the peptide/protein scale using a bottom-up proteomics approach. 
A

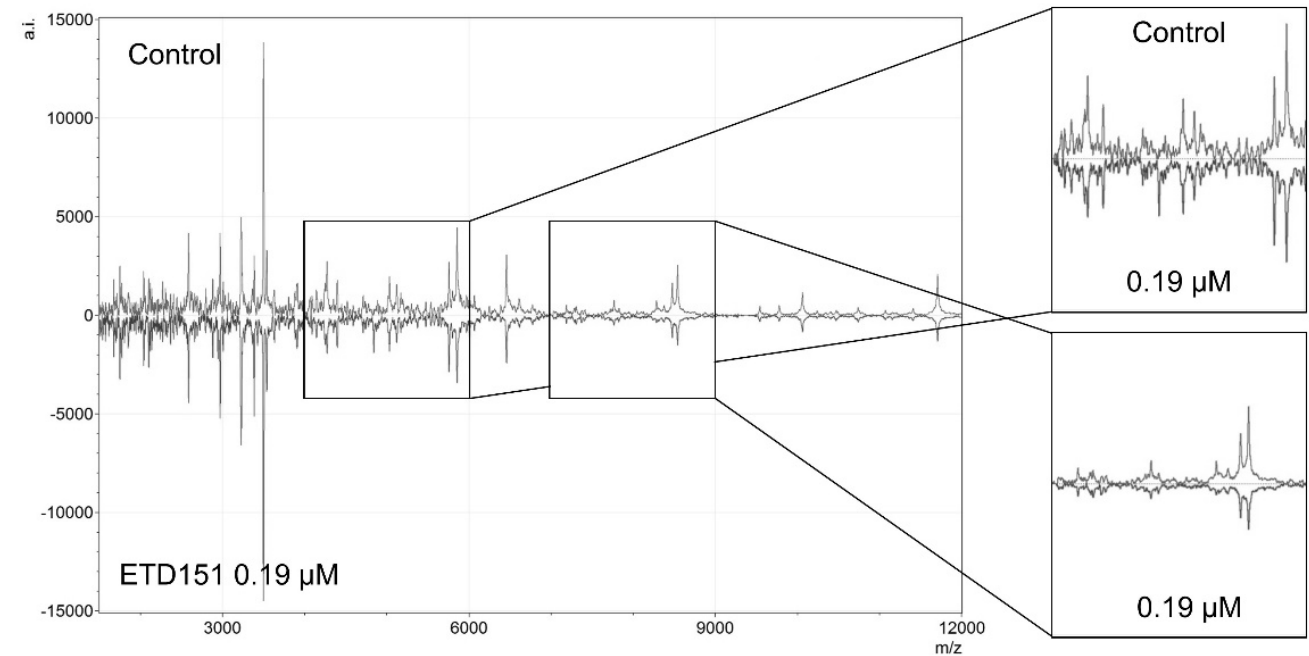

B

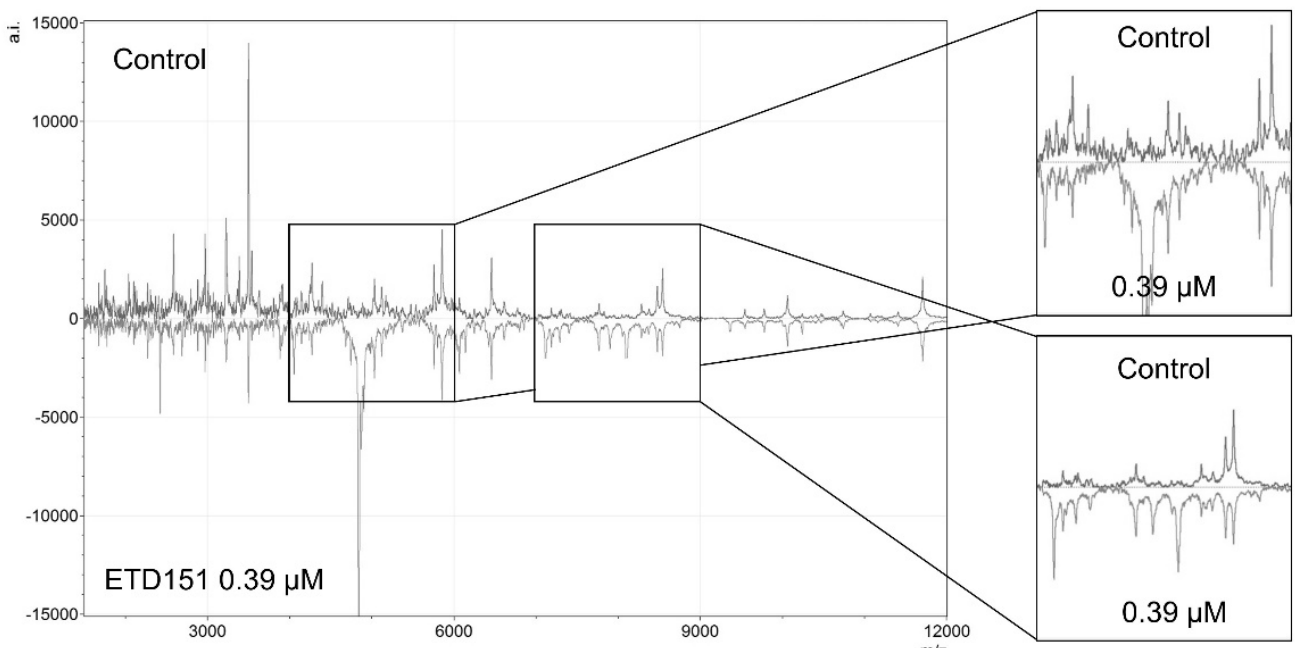

C

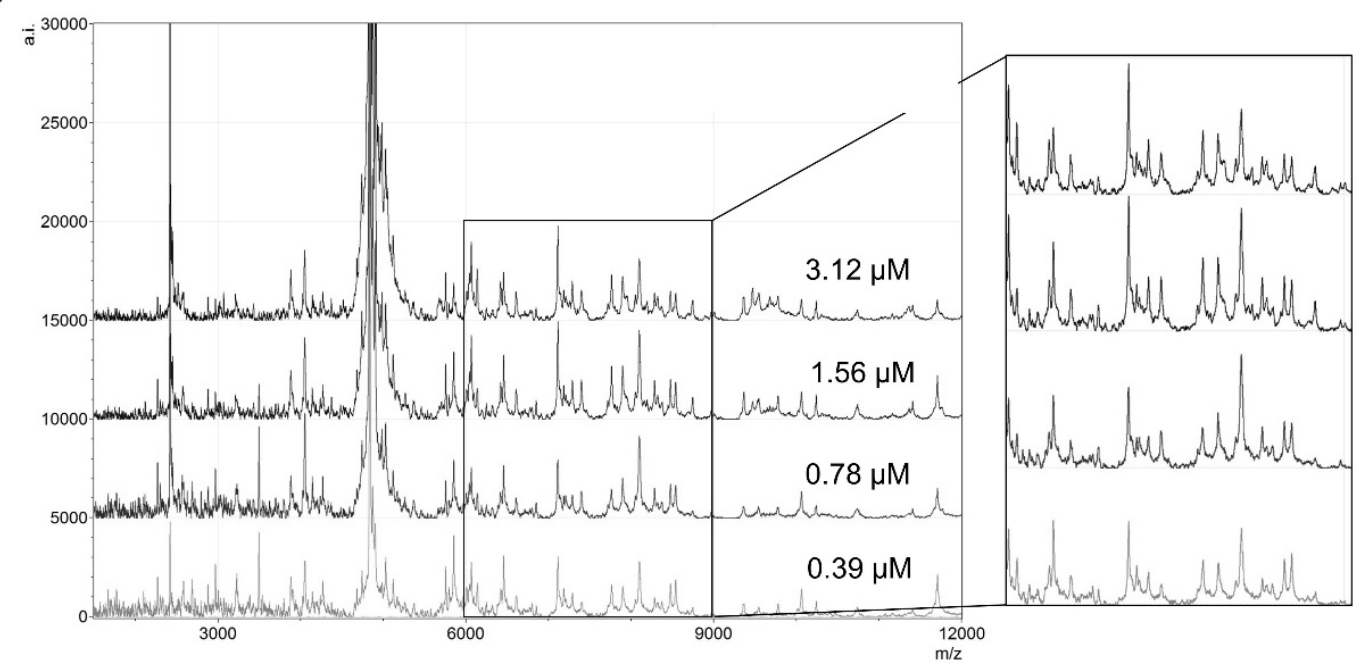

Figure 2: MALDI profiling of extracts of $B$. cinerea mycelia treated with ETD151 
457 The MALDI mass spectra show the average MALDI-molecular mass fingerprint $(n=3)$ from 458 mycelial protein extracts treated or not with the antifungal peptide ETD151. The mass spectra 459 are recorded in the $1,500-12,000$ mass $(\mathrm{m} / \mathrm{z})$ range, for untreated mycelia (Control) versus 460 mycelia treated with $0.19 \mu \mathrm{M}$ ETD151 (A) and $0.39 \mu \mathrm{M}$ ETD151 (B). Zooms show enlarged 461 area at the 4,000-6,000 and 7,000-9,000 mass ranges. The mass spectra recorded when the 462 mycelial cultures were treated with $0.39,0.78,1.56$ and $3.12 \mu \mathrm{M}$ ETD151 are also reported (C). 463 The zoom shows the enlarged mass range $(\mathrm{m} / \mathrm{z})$ between 6,000 and 9,000 . 
465 Differential proteomics analysis in response to ETD151

466 Our MALDI-MS results showed that the MFPs of the mycelial preparations are altered in 467 response to the different concentrations of ETD151 used to treat $B$. cinerea mycelia. To have a 468 better picture of the molecular response of $B$. cinerea mycelium upon our ETD151 treatment, we 469 used gel- and detergent-free proteomics to monitor protein changes on mycelial extracts. $B$. 470 cinerea mycelial peptide/protein acidic extracts prepared for MALDI-MS were dried under 471 vacuum prior to reduction-alkylation-enzymatic digestion. $B$. cinerea protein digests were then 472 analyzed by LC-ESI-MS/MS analysis as a conventional bottom-up proteomics approach. To 473 evaluate the methodology implemented and validate our data analysis strategy, two sets of 474 experiments were performed (Figure 3) allowing to generate two datasets (I and II). First, three 475 individual cultures were harvested, following the same experimental procedure, from the 476 collection of non-treated or treated cultures with different doses of ETD151 (Fig. 3A, dataset I). 477 Second, a triplicate experiment was conducted on three individual plates with three-day-old 478 mycelia (Fig. 3B, dataset II) originating from a unique culture of spores. 

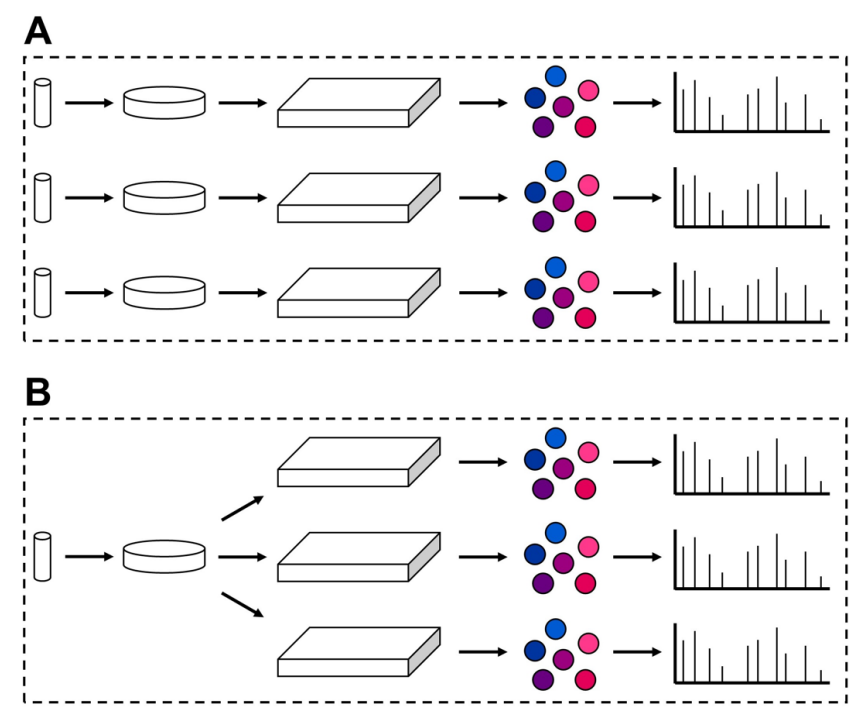

481 Fresh fungal material was obtained from a calibrated stock solution of $B$. cinerea spores 482 cultivated for seven days on a PDA medium. A fresh spore suspension was prepared and 483 distributed in a 96-well plate prior to incubation. Three-day-old mycelia were treated with water 484 (control experiment, blue) or with different concentrations of ETD151: $0.19 \mu \mathrm{M}$ (light blue), $4850.39 \mu \mathrm{M}$ (light pink), $0.78 \mu \mathrm{M}$ (pink), $1.56 \mu \mathrm{M}$ (light purple) and 3.12 $\mu \mathrm{M}$ (purple). After a 24h486 incubation, mycelia were collected and proteins were extracted. Samples were prepared for 487 bottom-up proteomics and digests analyzed by LC-ESI-MS/MS prior to data processing. (A) The 488 dataset I groups three individual cultures, while dataset II (B) is a triplicate experiment 489 corresponding to three individual plates coming from a unique culture of spores. 
Dataset I allowed the identification of 1,431 typical proteins with molecular masses ranging

491 from 4.8 to $466 \mathrm{kDa}$. Among these proteins, 1,430 originate from $B$. cinerea while the last one

492 corresponds to ETD151, detected by LC-ESI-MS/MS with $80 \%$ coverage (see raw data). We

493 observed an increase in the number of identified proteins in response to increasing

494 concentrations of ETD151 (Figure 4A, diamond plots). The average number (Fig. 4A, black

495 diamonds) of identified proteins was 532 and 534 for control and $0.19 \mu \mathrm{M}$ ETD151,

496 respectively. This average number increased to 794 and 899 proteins when the cultures were

497 treated with 0.39 and $3.12 \mu \mathrm{M}$ ETD151, respectively. The increase in the number of proteins

498 extracted in response to elevated concentrations of ETD151, may reflect a perturbation of the

499 integrity of the cell membrane of the mycelium and/or the stimulation of the expressions of

500 series of genes, and would corroborate microscopic data (see Fig. 1B) that points to an antifungal

501 effect of the defensin on $B$. cinerea. The reproducibility within dataset I was assessed for each

502 concentration of ETD151 as the average ratio between the number of identified proteins in each

503 experiment and the number of recurrent proteins (Fig. 4A, histograms), as identified in all three

504 experiments. The reproducibility within the control experiment and after a treatment with 0.19

$505 \mu \mathrm{M}$ ETD151was at $68 \pm 11 \%$ and $67 \pm 8 \%$, respectively. For $0.39 \mu \mathrm{M}$ ETD151, it was $73 \pm 6$

$506 \%$, and for higher concentrations of ETD151, the reproducibility was $75 \%$ with a standard

507 deviation $\leq 6 \%$ (Fig. 4A, square plots). Furthermore, a principal component analysis (PCA) was

508 run on proteins of dataset I and showed a clustering of experiments according to the

509 concentration of ETD151 (Figure 4B). Control and $0.19 \mu \mathrm{M}$ samples were clearly separated from

510 the other tested concentrations by the first component (42.8\%). The first component (PC1, Fig.

511 4B) may reflect the molecular impact of ETD151 on the B. cinerea mycelium. Samples from

5120.39 to $3.12 \mu \mathrm{M}$ of ETD151 could not be separated according to the second component (13.4\%). 
A

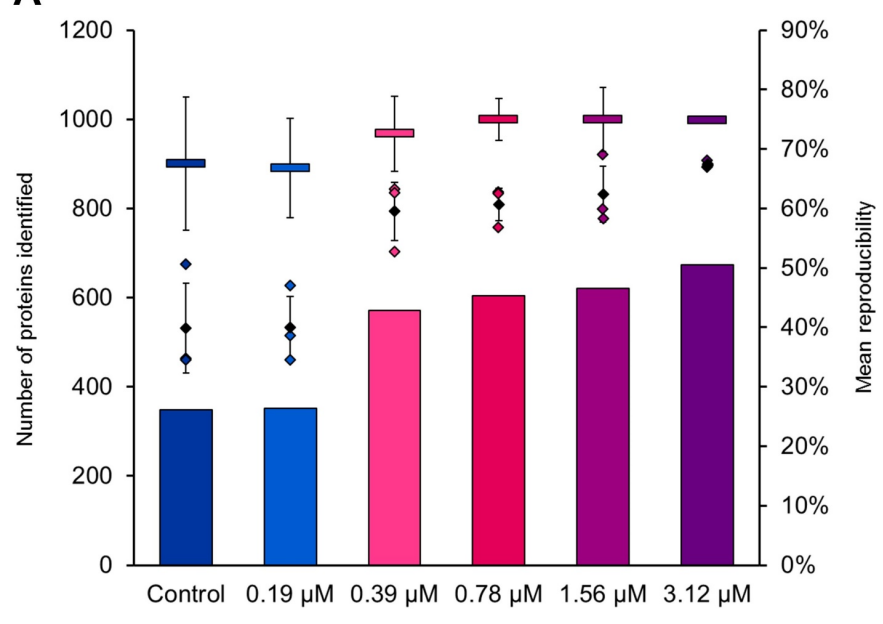

C

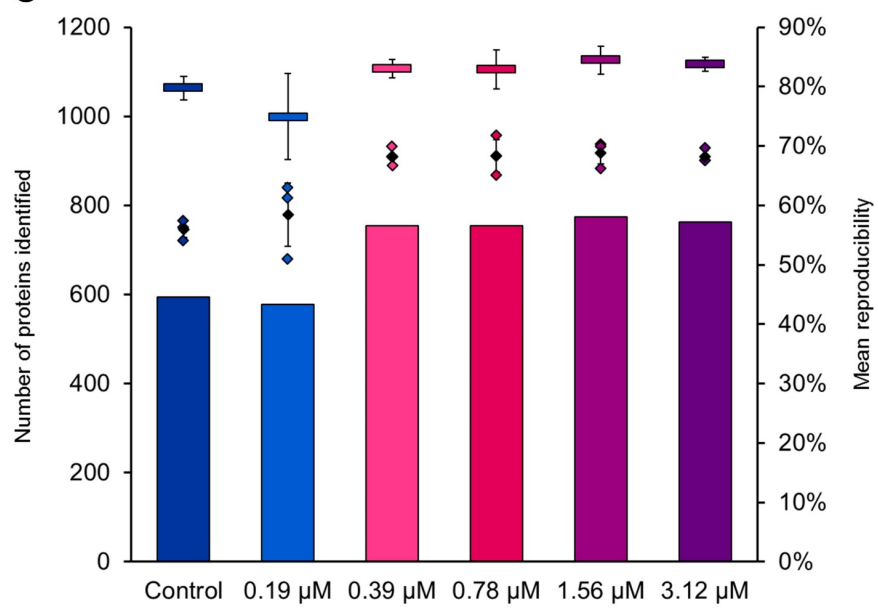

B
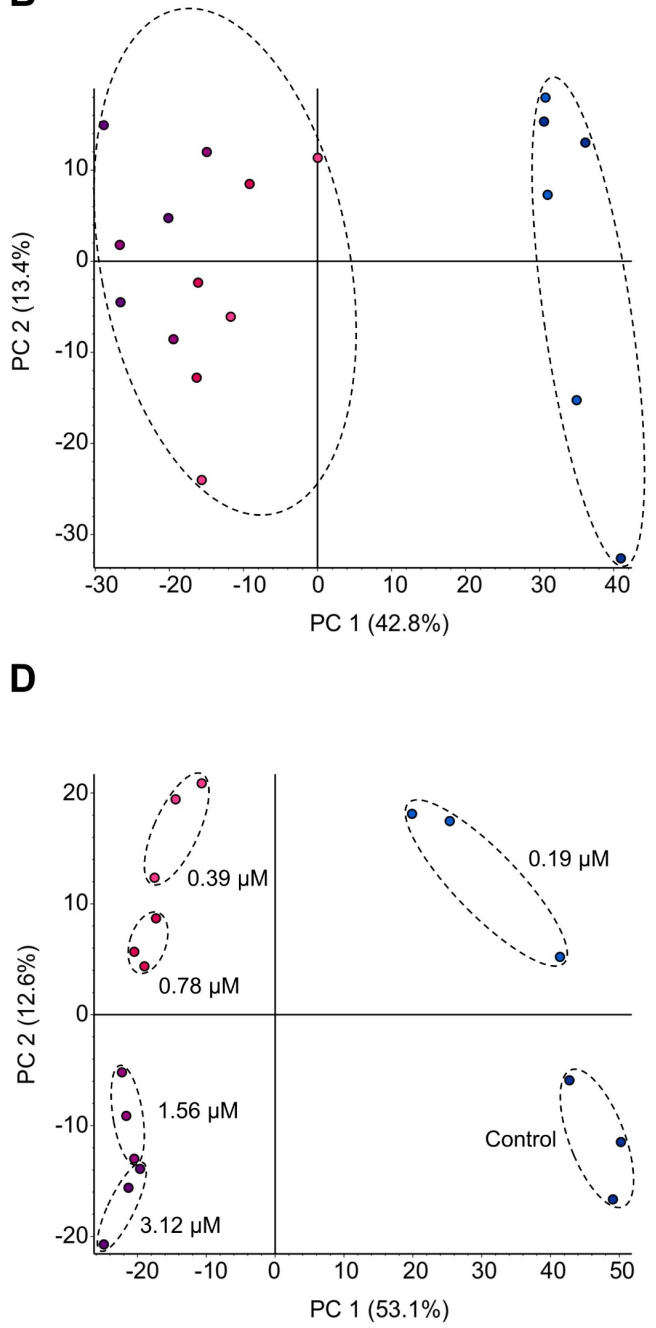

514 Figure 4: Protein identification of $\boldsymbol{B}$. cinerea mycelial extracts and assessment of reproducibility

516 (A) The number of proteins identified in samples of dataset $I$ is shown according to the 517 concentration of ETD151. Replicates and mean, along with the standard deviation, are 518 represented by colored and black diamond-shape plots, respectively. Histograms indicate the 519 number of recurrent proteins between all three replicates, the basis for calculation of 520 reproducibility. Mean reproducibility is represented by colored rectangles, along with the 521 standard deviation. (B) The Principal Component Analysis generated by Proteome Discoverer is 
522 shown for the 1,431 proteins identified within dataset I. Experiments are visualized according to 523 the concentration of ETD151 and the color code introduced previously. (C) The number of 524 proteins identified in samples of dataset II is reported according to the concentration of ETD151. 525 Values are represented in the same way as dataset I, and data of the Principal Component 526 Analysis generated for the 1,468 proteins identified in dataset II are shown in (D). 
$528466 \mathrm{kDa}$ mass range. The goal of this experimental design was to estimate the impact of the

529 initial culture of spores or of the harvest method on the reproducibility of the data. It should be

530 noted that working simultaneously on three individual microplates significantly complexified the

531 experimental protocol. Treatment of mycelia with ETD151, as well as the manual collection of

532 samples from each microplate, should be completed within a very short period of time. As

533 reported previously for dataset I, the average number of identified proteins increased upon

534 treatment with ETD151 (Figure 4C). As with dataset I, we observed an increase in the number of

535 identified proteins in response to increasing concentrations of ETD151 (Fig. 4C, diamond plots).

536 The average numbers (Fig. 4C, black diamonds) of identified proteins were 745 and 778 for the

537 control and $0.19 \mu \mathrm{M}$ ETD151 samples, respectively. This average number increased to 910 and

538917 proteins when the cultures were treated with 0.39 and $3.12 \mu \mathrm{M}$ ETD151 (Fig. 4C, diamond

539 plots). The reproducibility was assessed in the same way as for dataset I (Fig. 4C, histograms). In

540 the control and $0.19 \mu \mathrm{M}$ samples, the reproducibility was $80 \pm 2 \%$ and $75 \pm 7 \%$, respectively.

541 For higher concentrations of ETD151, the reproducibility ranged from 83 to $84 \%$ with a

542 standard deviation $\leq 3 \%$. PCA was also run on dataset II (Figure 4D). As observed for dataset I,

543 the first component $(53.1 \%)$ separated Control and $0.19 \mu \mathrm{M}$ samples from the other

544 concentrations. Unlike the PCA on dataset I, the second component (12.6\%) allowed to separate

545 the different samples according to the concentrations of ETD151.

546 This new experimental design improved the robustness of the data as the average

547 reproducibility between dataset I and II increased by $9 \%$ while the variability between replicates

548 decreased, as reflected by the reduction in standard deviations. This explains the greater weight

549 in our PCA analyses of the first component, at $43 \%$ and $53 \%$ in datasets I and II, respectively. 
550 Interestingly, in terms of the average number of identified or recurrent proteins, the samples

551 treated with $0.19 \mu \mathrm{M}$ ETD151 showed values closer to the control experiments (see above),

552 which is supported by the clustering observed for control and $0.19 \mu \mathrm{M}$ ETD151 replicates on one

553 side within the first component in PCA whereas replicates for higher concentrations aggregated

554 on the other side. Although the results improved in dataset II, samples for $0.19 \mu \mathrm{M}$ ETD151

555 were the only ones with a reproducibility below $80 \%$ and a standard deviation $>5 \%$. This

556 concentration of ETD151 appeared as an inflection point in the molecular effect of the antifungal

557 peptide as the changes induced by ETD151 barely allow to distinguish control from $0.19 \mu \mathrm{M}$

558 samples. Finally, datasets I and II were grouped to consolidate data analysis. Indeed, both dataset

559 shared similar properties on the increase in identified and recurring proteins for identical

560 concentrations of ETD151, from 0.39 to $3.12 \mu \mathrm{M}$.

561 The protein distributions were represented on a multidimensional Venn diagram based on six-

562 experimental datasets (untreated vs treated with five increasing concentrations of ETD151)

563 (Figure 5). The Venn diagram is a simplified tool, free of statistical test, allowing to classify

564 proteins from large datasets and to highlight groups of interest in our study. The diagram we

565 obtained, allowed to represent the logical relations between the 1,592 summed proteins identified

566 in the two datasets I and II. Among these proteins, 42 were specifically found in the non-treated

567 samples and 21 to 26 of them specifically in each concentration of ETD151 (21 in $0.19 \mu \mathrm{M}, 22$

568 in $0.39 \mu \mathrm{M}, 24$ in $0.78 \mu \mathrm{M}$ and 26 in $1.56 \mu \mathrm{M})$. We found the most abundant number of specific

569 proteins (44) when the mycelial culture was treated at $3.12 \mu \mathrm{M}$, the highest concentration of

570 ETD151. Interestingly, as previously discussed, at the inflexion point, 82 proteins were found

571 mutually in the control experiments and in samples treated with $0.19 \mu \mathrm{M}$ ETD151. Overall, 676

572 proteins were observed to be common to all samples, 120 proteins were found in all treated 
573 samples, regardless of the concentration of ETD151, and 185 proteins were specifically found at 574 the concentrations of $0.39 \mu \mathrm{M}$ ETD151 and above.

575 Our results demonstrated that ETD151 affected the molecular composition of $B$. cinerea 576 mycelial samples and confirmed, over a wide mass range (4.8 - $466 \mathrm{kDa})$, the results presented 577 previously by MALDI-MS MFPs up to $18 \mathrm{kDa}$. This comparative approach allows us to build an 578 overview of the behavior of protein groups following treatment with the peptide and to select by 579 label-free quantification characteristic proteins and metabolic pathways altered upon treatment $580 \quad$ with ETD151. 


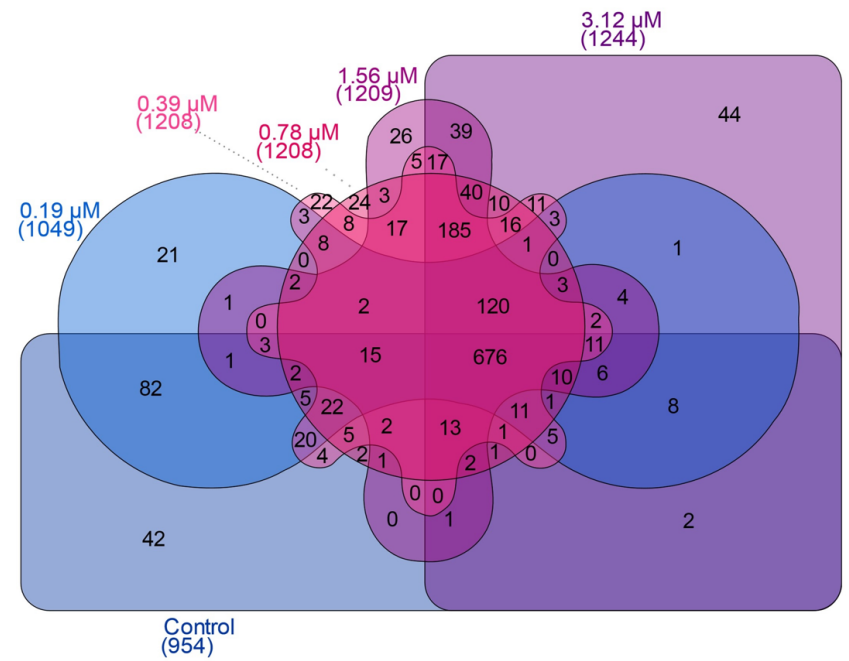

582 Figure 5: Multidimensional six-set Venn diagram

583 The Venn diagram generated on www.interactivenn.net displays the 1,592 summed proteins 584 from datasets I and II according to their presence or absence in mycelia treated with increasing 585 concentrations of ETD151:0.19 $\mu \mathrm{M}$ (light blue), $0.39 \mu \mathrm{M}$ (light pink), $0.78 \mu \mathrm{M}$ (pink), $1.56 \mu \mathrm{M}$ 586 (light purple) and $3.12 \mu \mathrm{M}$ (purple). 


\section{$587 \quad$ Label-free quantification analysis}

588 We identified 1,592 proteins and among them, 1,559 were quantified according to a label-free 589 approach. Abundance ratios and p-values were not calculated for 33 proteins. Significantly 590 dysregulated proteins were selected with volcano plots according to a p-value equal to 0.95 and a $591 \pm 2$-fold change (Supplementary data S4). Volcano plots are commonly used to display the 592 results of -omics experiments. This methodology allowed to highlight differentially abundant 593 proteins in the presence of different concentrations of ETD151 (Figure 6A). Increasing 594 concentrations of ETD151 of $0.19,0.39,0.78,1.56$ and $3.12 \mu \mathrm{M}$ resulted in the downregulation 595 of $23,56,66,73$ and 85 proteins, respectively (light grey); and in the upregulation of 86,173 , 596162,158 and 162 proteins, respectively (dark grey). Statistical analysis determined that out of the 597340 proteins identified, 134 were significantly downregulated, and 204 were significantly 598 upregulated (Figure 6B). Two proteins (Bcin02p06900, 40S ribosomal protein S20e, and 599 Bcin12p02600, putative mitochondrial protein), were downregulated in the presence of $0.19 \mu \mathrm{M}$ 600 of ETD151 and upregulated at higher concentrations. 
A

602

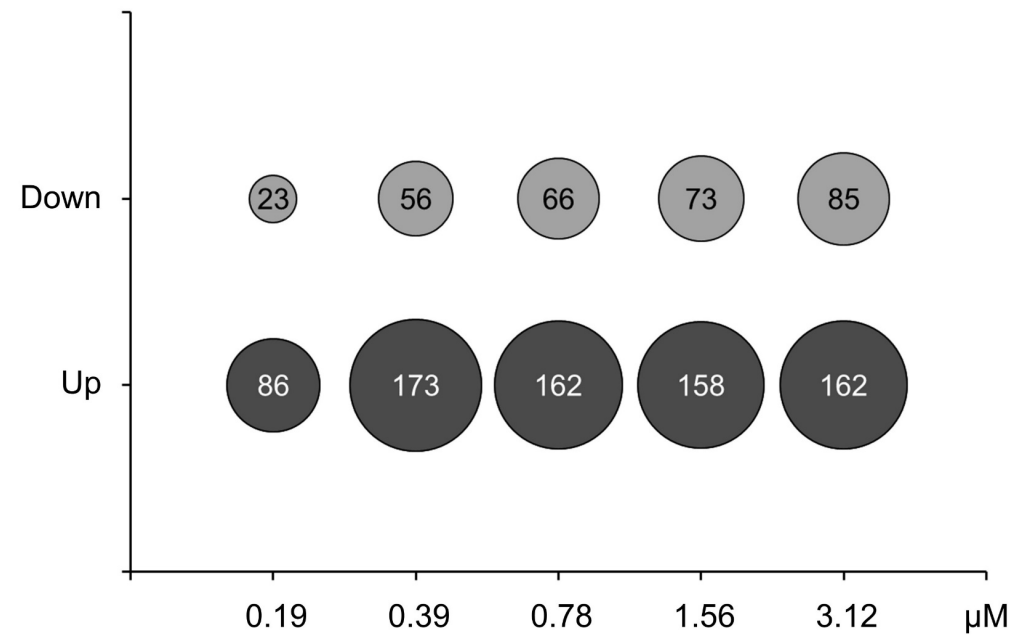

B

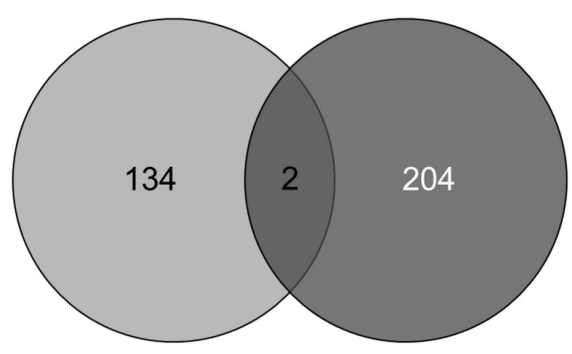

603 Figure 6: Significantly dysregulated proteins upon treatment of $\boldsymbol{B}$. cinerea mycelium with $604 \quad$ ETD151

605 (A) Bubble chart summarizes the differentially abundant proteins with a \pm 2 -fold change and a P606 value $\leq 0.05$ as detailed in volcano plots (see Supplementary data S4). (B) The Venn diagram 607 pooling the two sets of proteins modulated upon treatment with ETD151. A total of 340 unique 608 proteins are reported including 134 downregulated (light grey), 204 upregulated (dark grey) and 609 two that were both down- and upregulated (intersecting of the two sets).

610 


\section{$611 \quad$ Gene ontology and network analysis}

612 Proteins were annotated using ProteinCenter (Proteome Discoverer) to investigate molecular

613 functions, biological processes, and cellular components from the Gene Ontology (GO) database

614 (Figure 7). The molecular function-based categorization showed that among the 340 selected

615 proteins, $168(42 \%)$ could not be classified while the others were classified according to the GO

616 terms as follow: $103(26 \%)$ have catalytic activities (GO:0003824), 92 (23\%) have binding

617 properties (GO:0005488), $27(7 \%)$ contribute to the structural integrity of a complex or its

618 assembly within or outside a cell (GO:0005198), 7 (2\%) act as transporters (e.g. lipids, proteins,

619 oxygen) (GO:0005215), 4 (1\%) are regulators of molecular functions (GO:0098772), and finally

$6202(0.5 \%)$ were recognized as having antioxidant activity (GO:0016209) (Fig. 7A). Following the

621 biological process-based categorization, 178 (49\%) among the 340 proteins could not be

622 classified. The most affected process is metabolism with 125 proteins (34\%) (GO:0008152). The

623 rest of the proteins are involved in biological processes such as (i) localization (21, 6\%)

624 (GO:0051179), (ii) regulation $(15,4 \%)$ (GO:0065007), (iii) cellular component organization (14, $6254 \%)(\mathrm{GO}: 0071840)$, (iv) response to a stimulus $(7,2 \%)(\mathrm{GO}: 0050896)$, or (v) cellular process $(2$,

626 1\%) (GO:0009987) (Fig. 7B). Cellular component annotation was also investigated despite a 627 lack of information for B. cinerea in this GO categorization. Among the 340 selected proteins, $628288(84 \%)$ could not be classified (data not shown). 
A: Molecular function

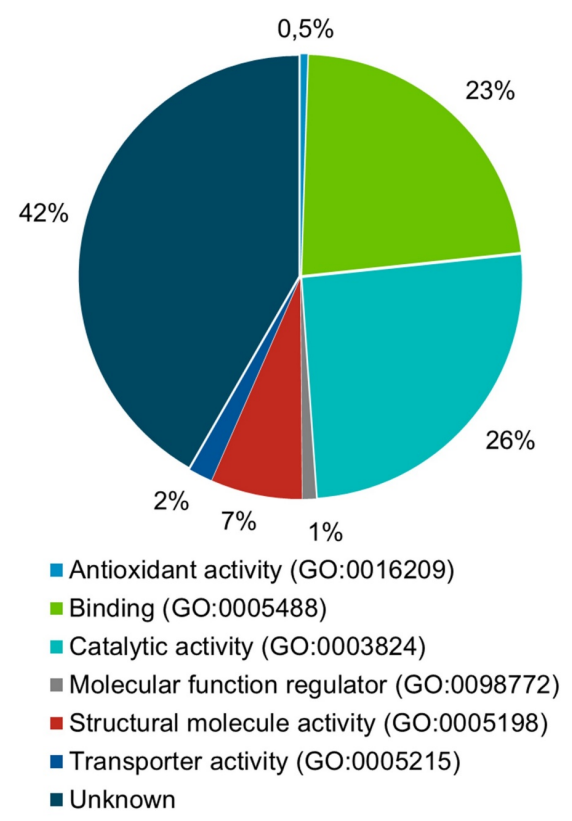

B: Biological process

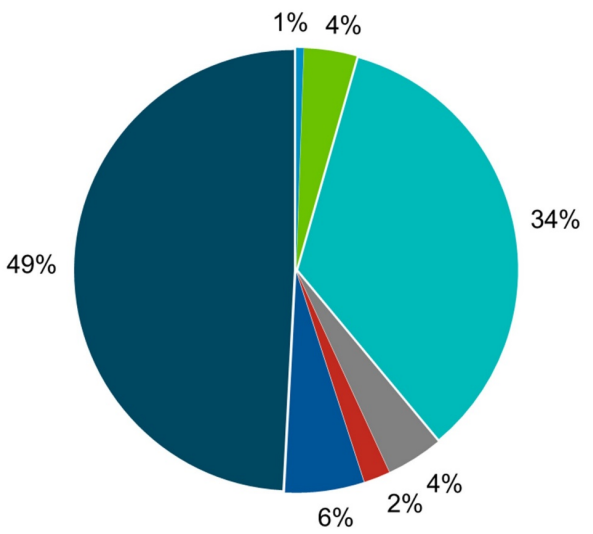

- Cellular process (GO:0009987)

n Cellular component organization (GO:0071840)

- Metabolic process (GO:0008152)

niological regulation (GO:0065007)

- Response to stimulus (GO:0050896)

- Localization (GO:0051179)

- Unknown

632 Proteins selected (340) according to their differential abundance are categorized according to

633 their (A) molecular function and (B) role in biological processes. 


\section{Molecular impact of ETD151 on B. cinerea pathways}

635 Significantly modulated proteins upon treatment with ETD151 were searched in Botrytis 636 cinerea KEGG pathways (117 pathways reported). From the 339 proteins (340 minus ETD151)

637 previously selected, 106 were successfully mapped over 67 different pathways with a varied 638 number of hits, from 1 to 38 matched proteins per map (Supplementary data S5). Pathways with 639 less than five hits were not considered for further investigations, as a low number of matched 640 proteins did not allow us to interpret their relevance in the mechanism of action of ETD151 on

641 B. cinerea. Moreover, most of these maps were included in carbohydrate, lipid or amino acid 642 metabolisms. Direct effects on these pathways had been evaluated by measuring the uptake and 643 incorporation of radiolabeled precursors $\left({ }^{14} \mathrm{C}\right.$-glucose, ${ }^{3} \mathrm{H}$-phenylalaline and ${ }^{14} \mathrm{C}$-acetate) by 644 Botrytis cinerea spores in the presence of ETD151 (data not shown). ETD151 did not interfere 645 with carbohydrate, protein and lipid biosynthesis confirming that modulated proteins in 646 previously mentioned pathways resulted from global stress in response to an antifungal 647 molecule. The following global pathway maps (metabolic pathways (bfu01100), biosynthesis of 648 secondary metabolites (bfu1110), biosynthesis of antibiotics (bfu01130) and carbon metabolism 649 (bfu01200) were also excluded from the analysis in favor of the study of specific maps. By 650 considering the filters previously described, six pathways were impacted by the treatment, 651 namely ribosome (bfu03010), spliceosome (bfu03040), protein processing in endoplasmic 652 reticulum (bfu04141), endocytosis (bfu04144), MAPK signaling pathway (bfu04011), and 653 oxidative phosphorylation (bfu00190). 
656 Ribosomes are essentially very complicated nano-factories for producing proteins. In our

657 study, 80 proteins, out of a total of 103 reported in this pathway for $B$. cinerea, were identified in 658 datasets I and II (Supplementary data S6). Upon treatment with ETD151, 25 ribosomal proteins 659 were significantly modulated. Starting at a concentration of $0.39 \mu \mathrm{M}$ of ETD151 this included 24 660 up-regulated proteins, as eight in the small subunit 40S: Bcin01p00980/S2e, 661 Bcin02p06900/S20e, Bcin03p05970/S9, Bcin03p06990/S16e, Bcin03p07110/S15Ae, 662 Bcin03p07120/S12e, Bcin07p00830/S13 and Bcin08p00760/S29e, and 16 in the large subunit 663 60S: Bcin01p09660/L35Ae, Bcin03p01900/L39e, Bcin03p06530/L7Ae, Bcin03p07630/L7e, 664 Bcin05p01600/L18Ae, Bcin05p06070/L38e, Bcin07p00520/L27e, Bcin08p03200/L4, 665 Bcin09p03550/L14e, Bcin09p05600/L30e, Bcin10p04290/L17e, Bcin10p04590/L12e, 666 Bcin14p04100/L22e, Bcin08p02490/L32e (starting from $0.19 \mu \mathrm{M}$ ), Bcin14g04790/L15e 667 (starting from $0.19 \mu \mathrm{M}$ ) and Bcin08p00590/L15 (at 0.39 and $0.78 \mu \mathrm{M}$ ). Bcin14p02830, 60S 668 ribosomal protein $\mathrm{L} 3 \mathrm{e}$, is the only downregulated protein, starting from the concentration of 669 ETD151 of $0.39 \mu \mathrm{M}$. To our knowledge, there is no report in the literature concerning antifungal 670 insect or plant defensins directly targeting protein biosynthesis in ribosomes. The modulation of 671 ribosome-related subunits might reflect an attempt to keep protein synthesis at optimal levels as 672 the presence of the antifungal defensin ETD151 disturbs protein biosynthesis and degradation.

$674 \quad$ Effect on the spliceosome

675 The spliceosome is a dynamic complex, located primarily within the nucleus of eukaryotic 676 cells, and plays an essential role in the maturation process of mRNA. In our study, 27 proteins, 677 out of a total of 84 reported in this pathway for B. cinerea, were identified (Supplementary data 
678 S6). Upon treatment with ETD151, 7 proteins were significantly dysregulated.

$679 \mathrm{Bcin} 08 \mathrm{p} 03160 / \mathrm{Smd} 2$ and $\mathrm{Bcin} 08 \mathrm{p} 06600 / \mathrm{Smd} 3$, small nuclear ribonucleoproteins, and

680 Bcin09p07050/Lsm7, an snRNA-associated Sm-like protein, were upregulated at $0.39 \mu \mathrm{M}$ of

681 ETD151 and higher concentrations. In contrast, four proteins were downregulated in the

682 presence ofETD151: Bcin09p06610/PPIL1 peptidyl-prolyl cis-trans isomerase-like from 0.19

$683 \mu \mathrm{M}, \mathrm{Bcin} 08 \mathrm{p} 00660 / \operatorname{Prp} 1$ pre-mRNA-processing factor from $1.56 \mu \mathrm{M}$, Bcin09p06940/SF3b

684 splicing factor and Bcin13p05240/THOC THO complex subunit at $3.12 \mu \mathrm{M}$. There is no clear

685 tendency to interpret the molecular changes induced by ETD151 on this pathway. Moreover, as

686 there is no report in the antifungal defensin literature of a direct effect on the spliceosome, this

687 pathway was not considered for the continuation of the work on the mechanism of action of

688 ETD151.

689

690 Effect on protein processing in the endoplasmic reticulum (ER)

691 The ER is a continuous membrane system found within the cytoplasm of eukaryotic cells 692 allowing for protein synthesis, folding, modification and in some cases transport to the Golgi 693 apparatus through vesicles. In our study, 37 proteins, out of a total of 70 reported in this pathway

694 for B. cinerea, were identified (Supplementary data S6). Upon treatment with ETD151, seven 695 proteins were significantly dysregulated. Bcin13p00700/ERGIC53 and Bcin05p05180/OS9 are 696 upregulated starting a concentration of $0.39 \mu \mathrm{M}$. Bcin07p01430/Sec62, Bcin02p05070/Sec24, 697 Bcin01p05490/Bcnpl4 and Bcin10p00510 are downregulated starting at $0.39 \mu \mathrm{M}$ of ETD151. 698 These proteins play roles in the transport of proteins from the ER. Proteins that are located in the 699 ER and/or Golgi apparatus, are interesting to highlight, as sphingolipids, including 700 glucosylceramides, fungal partners of RsAFP2" and possibly ETD151, are synthesized in the ER 
701 and Golgi apparatus ${ }^{2}$. Further investigation will be necessary to monitor a direct effect of

702 ETD151 on glucosylceramide biosynthesis and/or export.

703

$704 \quad$ Effect on endocytosis

705 Endocytosis is a cellular process in which substances (e.g. nutrients, plasma membrane

706 proteins, lipids) are brought into the cell. Interestingly, research on antifungal plant defensins

707 demonstrated that certain ones, such as MtDef4, Psd1 and NaD1, can be internalized by fungal

708 cells upon interaction with a membrane partner ${ }^{2}$. Mechanisms of fungal cell entry are still under

709 investigation, however it is known that MtDef4 and NaD1 internalization are energy dependent

710 and require endocytosis in Neurospora crassa $^{33}$ and Candida albicans ${ }^{\text {s }}$, respectively. In our

711 study, 35 proteins, out of a total of 58 reported in this pathway for B. cinerea, were identified

712 (Supplementary data S6). Upon treatment with ETD151, 7 proteins were significantly

713 dysregulated. Three of them were upregulated in the presence of ETD151:

714 Bcin13p05610/CAPZA capping protein (actin filament) muscle Z-line starting at a concentration

715 of $0.19 \mu \mathrm{M}, \mathrm{Bcin} 11 \mathrm{p} 01640 / \mathrm{VPS} 26$ and Bcin11p01920/VPS35 vacuolar protein sorting-

716 associated protein at a concentration of $0.39 \mu \mathrm{M}$. In contrast, four were downregulated:

717 Bcin01p06150/Arp2/3 actin related protein $2 / 3$ complex subunit, at a concentration of $0.19 \mu \mathrm{M}$

718 only, Bcin03p0497/Epsin membrane protein and Bcin13p04030/ArfGAP stromal membrane-

719 associated protein starting at the concentration of $0.39 \mu \mathrm{M}$, and Bcin13p00990/Hrs hepatocyte

720 growth factor-regulated tyrosine kinase substrate at the concentrations of 0.78 and $1.56 \mu \mathrm{M}$.

721 These results could be valuable for future research on the mechanism of action of ETD151, and

722 specifically the behavior of the antifungal defensin upon its interaction with the fungal 723 membrane. 
$725 \quad$ Effect on MAPK signaling pathway

726 Fungi, like other eukaryotes, rely on the rapid transduction of signals through the mitogen-

727 activated protein kinase (MAPK) pathway in order to overcome environmental stresses such as

728 the addition of chemical fungicides and antifungal peptides. At lower concentrations, one or

729 more stress response pathways are activated to respond to the cell damage. At higher

730 concentrations, the fungal stress response leads to the inhibition of cell growth or induction of

731 cell death. Several antifungal plant defensins ${ }^{34}$ (e.g. RsAFP2, Psd1, MsDef1) are known to

732 activate the MAPK pathway, with the cell wall integrity pathway (CWI), inducing changes to

733 cell wall biogenesis, $\beta$-glucan synthesis and the organization of the actin cytoskeleton. The

734 Defensin NaD1 activates the high-osmolarity glycerol (HOG) pathway, leading to an increase in

735 intracellular glycerol which provides protection against osmotic stress. In our study, 23 proteins,

736 out of a total of 56 reported in the HOG pathway (bfu04011) for B. cinerea, were identified in

737 datasets I and II (Supplementary data S6). Upon treatment with ETD151, three proteins were

738 dysregulated in the CWI pathway: Bcin08p03780/Rom1,2 (down at $3.12 \mu \mathrm{M}$ ),

739 Bcin02p06930/Fks2 (up starting from $0.19 \mu \mathrm{M}$ ) and Bcin16p00490 (up starting from $0.19 \mu \mathrm{M}$ );

740 one protein was dysregulated in the HOG pathway: Bcin16p04710 (down starting from 0.39

$741 \mu \mathrm{M})$. The protein Bcin16p00490/Cdc28, in both CWI and HOG pathways, was also upregulated

742 starting from $0.19 \mu \mathrm{M}$.

743

$744 \quad$ Effect on oxidative phosphorylation

745 Oxidative phosphorylation, coupling the cascade of oxidation-reduction of the mitochondrial 746 respiratory chain and the phosphorylation of ADP to ATP, is an essential metabolic pathway for 
747 the survival of most aerobic eukaryotic organisms. In our study, 60 out of a total of 72 reported

748 proteins in the B. cinerea bfu00190 pathway were identified (Figure 8A). Upon treatment with

749 ETD151, two subunits of NADH dehydrogenase (complex I) Bcin08p06780/NDUFV1,

750 Bcin08p01490/NDUFA8 were significantly up-regulated starting from a concentration of

$7510.78 \mu \mathrm{M}$ of ETD151. Similarly, a subunit of succinate dehydrogenase (complex II)

752 Bcin05p04430 was also up-regulated at $0.19 \mu \mathrm{M}$ of ETD151. By contrast, a subunit of

753 ubiquinol-cytochrome c reductase (complex III) Bcin03p08370/QCR9 was significantly down-

754 regulated at $0.19 \mu \mathrm{M}$ of ETD151. Finally, several subunits of F-type H+-transporting ATPase

755 (complex V) were significantly modulated upon treatment: Bcin02p02510/epsilon (down at 0.19

$756 \mu \mathrm{M}$ ), Bcin10p01500/c (up starting at $0.39 \mu \mathrm{M}$ ), Bcin02p02250/e (down at $3.12 \mu \mathrm{M}$ ) and

757 Bcin12p02610/k (down starting at $1.56 \mu \mathrm{M}$ ). Our proteomics data suggested a mitochondrial

758 dysfunction in the presence of the antifungal defensin ETD151. To go further, the direct activity

759 of ETD151 on complexes I to III of the mitochondrial respiratory chain was assessed and

760 compared to boscalid and antimycin, two molecules providing reference profiles as inhibitors of

761 succinate dehydrogenase and ubiquinol-cytochrome c reductase, respectively (Figure 8B). A

762 colorimetric assay based on the reduction of ferricyanide allowed to measure combined activities

763 of complexes I and III on the one hand, and complexes II and III on the other hand. The activity

764 of boscalid demonstrated a partial inhibition (50\%), when testing complexes I and III together,

765 and a total inhibition, when testing complexes II and III together. The activity of antimycin A

766 was characterized by a total inhibition in both associations of complexes. In the presence of

767 ETD151, no inhibition was measured regardless of the tested complex, using up to $50 \mu \mathrm{M}$ of the

768 antifungal defensin. This result confirmed that ETD151 does not interact directly with complexes

769 of the mitochondrial respiratory chain. Mitochondrial dysfunction highlighted by our proteomics 
770 data may result from mitochondrial membrane perturbation, reflecting a secondary effect of 771 ETD151 disturbing cell membrane integrity. 
A

OXIDATIVE PHOSPHORYLATION

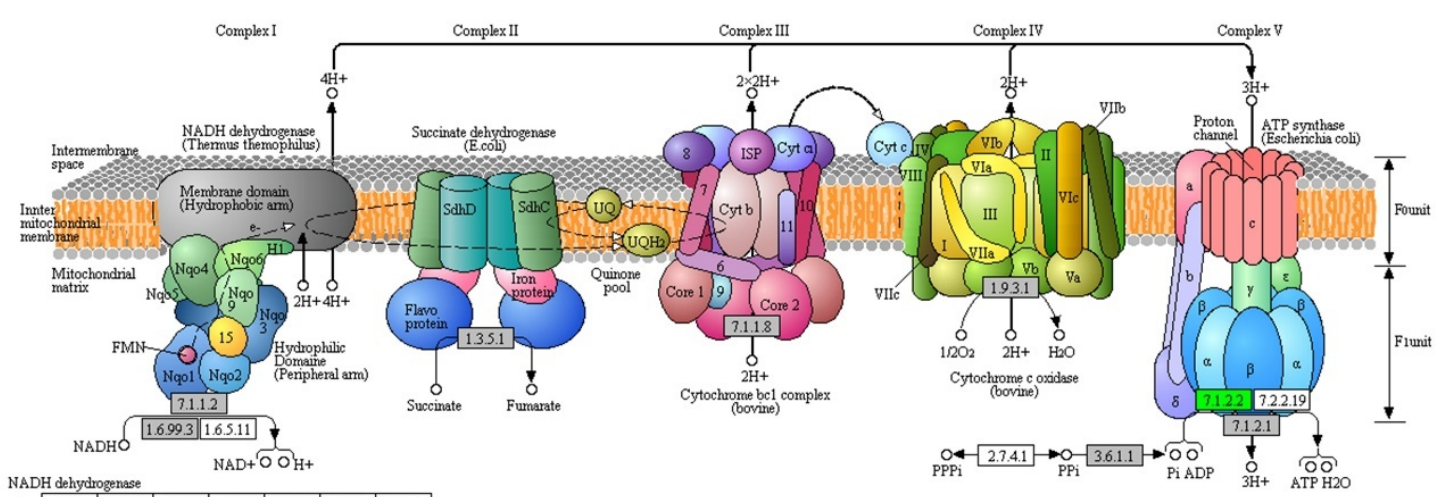

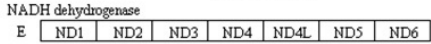

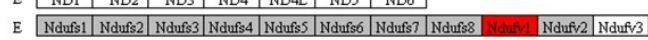

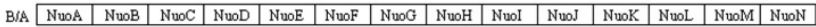

B/A \begin{tabular}{|l|l|l|l|l|l|l|l|l|l|l|l|l|l|l|l|l|}
\hline NdhC & NdhK & NdhJ & NdhH & NdhA & NdhI & NdhG & NdhE & NdhF & NdhD & NdhB & NdhL & NdhM & NdhN & HoxE & Hoxf & HoxU \\
\hline
\end{tabular}

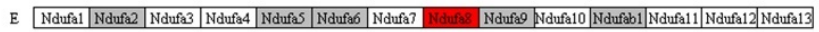

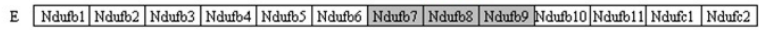

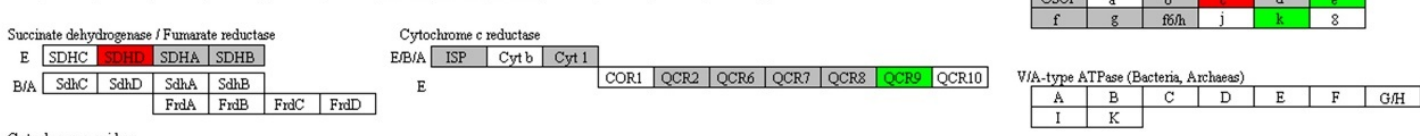

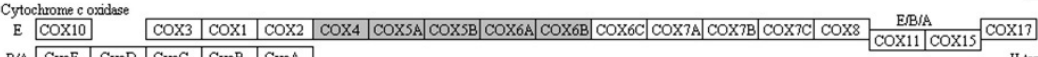

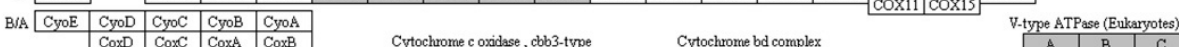

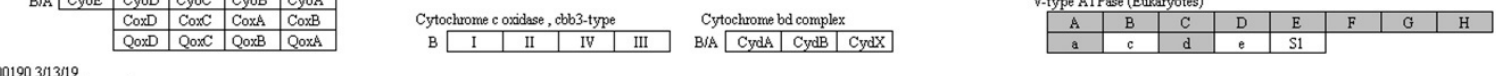
00190 $3 / 131199$
(c) Kanehisa Laboratones

B

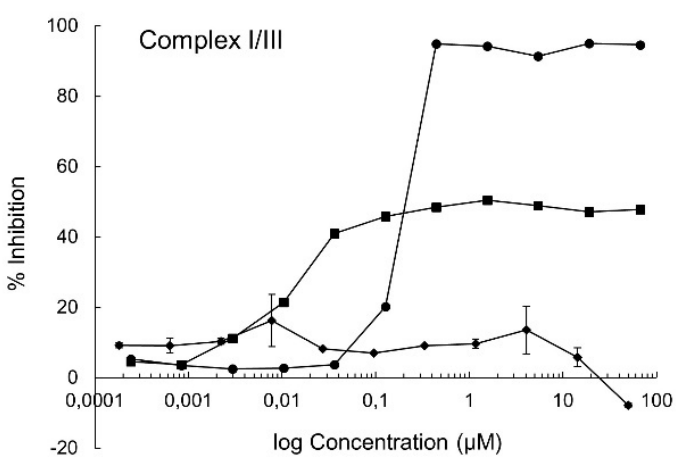

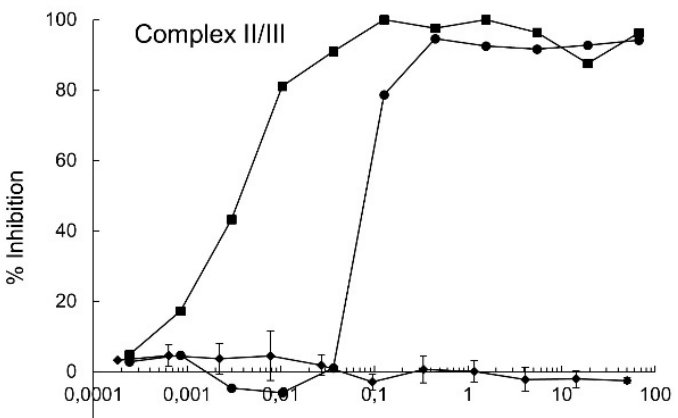

$\log$ Concentration $(\mu \mathrm{M})$

773 Figure 8: Effect of ETD151 on the B. cinerea mitochondrial chain respiration complex

774 (A) Mapping of dysregulated proteins upon treatment with ETD151 on the oxidative 775 phosphorylation pathway (bfu00190) from the KEGG database. For B. cinerea, 72 proteins are 776 reported on this map of which 60 were identified in this study (grey). Eight differentially

777 abundant proteins were found, upregulated (red) and downregulated (green). (B) Activity of

778 ETD151 (diamond), boscalid (square) and antimycin A (circle) on complex I/III and complex 
$779 \mathrm{II} / \mathrm{III}$ of $B$. cinerea mitochondria. Results for ETD151 are expressed as the average of a duplicate 780 experiment along with standard deviation. 


\section{Conclusions \& Perspectives}

782 Through this study, we demonstrated that the proposed approach offers an experimental

783 workflow to evaluate changes in the peptide and protein contents of mycelial extracts of $B$.

784 cinerea in response to different concentrations of the antifungal peptide ETD151. As an initial

785 approach, molecular mass fingerprinting by MALDI-MS allowed to highlight molecular changes

786 in B. cinerea mycelia treated with ETD151. Even with spectra obtained on a partial mass range

$787(<20 \mathrm{kDa})$, it could be interesting to compare average fingerprints in the presence of ETD151 to

788 spectra generated with other antifungal defensins. This comparison could direct the study on the

789 mechanism of action of ETD151 toward other peptides. As a second step, bottom-up analyses of

790 mycelial extracts provided robust and reproductible data, necessary to study in-depth molecular

791 changes induced by ETD151 on $B$. cinerea. Thus, we revealed six pathways (ribosome

792 (bfu03010), spliceosome (bfu03040), protein processing in endoplasmic reticulum (bfu04141),

793 endocytosis (bfu04144), MAPK signaling pathway (bfu04011), and oxidative phosphorylation

794 (bfu00190)) impacted by the insect defensin. At least for one of these pathways, we also

795 demonstrated that the observed impact must be indirect. Indeed, we clearly demonstrated that

796 there was no direct effect of ETD151 on the $B$. cinerea mitochondrial respiration chain. As a

797 conclusion, proteomics could offer a way to improve the research strategy into the resolution of

798 antifungal mechanisms of action by targeting preferentially involved proteins or metabolic

799 pathways. Finally, an additional objective for a deeper understanding of the mechanisms of

800 action of ETD151 is to study the interaction of ETD151 with lipid components of the fungal

801 membrane, such as e.g. glucosylceramides.

802 


\section{Supporting Information}

804 The following files are available free of charge.

805 S1: Composition of reaction mix for the activity test on respiratory chain complex

806 S2: MALDI-MS fingerprinting of $B$. cinerea mycelial extracts

807 S3: Distribution of MALDI-MS ions in B. cinerea mycelium fingerprints

808 S4: Volcano plots depicting significantly dysregulated B. cinerea proteins in the presence of

$809 \quad$ ETD151

810 S5: List of KEGG B. cinerea pathways with significantly dysregulated proteins

811 S6: Mapping of B. cinerea dysregulated proteins on pathways impacted upon treatment with

$812 \quad$ ETD151

813

\section{Author Contributions}

815 Idea and design of the study: PB, CL, TK.

816 Mass spectrometric analysis: TA, PB, SV.

817 Pathway analysis: TA, TK, PB.

818 Statistical analysis: TA, TK, PB, SV.

819 Drafting the manuscript: TA, PB, CL, TK.

820 Review of manuscript for important intellectual content: all authors. 
823 The authors declare no conflicts of interest.

$825 \quad$ Notes

826 All relevant data are within the paper and its Supporting Information files.

\section{Acknowledgments \& Funding Sources}

828 This work was supported by the Convention Industrielle de Formation par la Recherche CIFRE $829 n^{\circ} 2015 / 1111$ - through the research contract between Bayer SAS, the French National Centre for

830 Scientific Research (CNRS, CBM UPR 4301 and IAB UMR 5309, France) and Research and

831 Development from the Association Plateforme BioPark of Archamps (France). We thank our

832 colleagues: Dr Karim Arafah and Victor Masson, in the MassOmics group of the Association

833 Plateforme BioPark of Archamps, for providing knowledge in LC-ESI-MS/MS and data

834 processing, and in the preparation and processing of mycelial extracts, respectively. We also

835 thank Stephane Peyrard and Catherine Sirven from Computational Life Science group in the La

836 Dargoire Research Center (Bayer SAS, Lyon), for providing their expertise in protein annotation 837 and data processing, respectively.

\section{References}

(1) Pennisi, E. Armed and Dangerous. Science 2010, 327 (5967), 804-805. https://doi.org/10.1126/science.327.5967.804.

844

(2) Fisher, M. C.; Henk, D. A.; Briggs, C. J.; Brownstein, J. S.; Madoff, L. C.; McCraw, S. L.; Gurr, S. J. Emerging Fungal Threats to Animal, Plant and Ecosystem Health. Nature 2012, 484 (7393), 186-194. https://doi.org/10.1038/nature10947. 
(3) Williamson, B.; Tudzynski, B.; Tudzynski, P.; van Kan, J. A. L. Botrytis Cinerea: The Cause of Grey Mould Disease. Mol. Plant Pathol. 2007, 8 (5), 561-580. https://doi.org/10.1111/j.1364-3703.2007.00417.x.

(4) van Kan, J. A. L. Licensed to Kill: The Lifestyle of a Necrotrophic Plant Pathogen. Trends Plant Sci. 2006, 11 (5), 247-253. https://doi.org/10.1016/j.tplants.2006.03.005.

(5) Dean, R.; van Kan, J. A. L.; Pretorius, Z. A.; Hammond-Kosack, K. E.; Pietro, A. D.; Spanu, P. D.; Rudd, J. J.; Dickman, M.; Kahmann, R.; Ellis, J.; et al. The Top 10 Fungal Pathogens in Molecular Plant Pathology. Mol. Plant Pathol. 2012, 13 (4), 414-430. https://doi.org/10.1111/j.1364-3703.2011.00783.x.

(6) Hahn, M. The Rising Threat of Fungicide Resistance in Plant Pathogenic Fungi: Botrytis as a Case Study. J. Chem. Biol. 2014, 7 (4), 133-141. https://doi.org/10.1007/s12154-0140113-1.

(7) Lucas, J. A.; Hawkins, N. J.; Fraaije, B. A. Chapter Two - The Evolution of Fungicide Resistance. In Advances in Applied Microbiology; Sariaslani, S., Gadd, G. M., Eds.; Academic Press, 2015; Vol. 90, pp 29-92.

(8) Botrytis - the Fungus, the Pathogen and Its Management in Agricultural Systems; Fillinger, S., Elad, Y., Eds.; Springer International Publishing: Cham, 2016.

(9) Brown, K. L.; Hancock, R. E. Cationic Host Defense (Antimicrobial) Peptides. Curr. Opin. Immunol. 2006, 18 (1), 24-30. https://doi.org/10.1016/j.coi.2005.11.004.

(10) Cornet, B.; Bonmatin, J.-M.; Hetru, C.; Hoffmann, J. A.; Ptak, M.; Vovelle, F. Refined Three-Dimensional Solution Structure of Insect Defensin A. Structure 1995, 3 (5), 435 448. https://doi.org/10.1016/S0969-2126(01)00177-0.

(11) Zhu, S.; Gao, B.; Tytgat, J. Phylogenetic Distribution, Functional Epitopes and Evolution of the CS $\alpha \beta$ Superfamily. Cell. Mol. Life Sci. CMLS 2005, 62 (19-20), 2257-2269. https://doi.org/10.1007/s00018-005-5200-6.

(12) Shafee, T. M. A.; Lay, F. T.; Hulett, M. D.; Anderson, M. A. The Defensins Consist of Two Independent, Convergent Protein Superfamilies. Mol. Biol. Evol. 2016, 33 (9), 23452356. https://doi.org/10.1093/molbev/msw106.

(13) Vriens, K.; Cammue, B. P. A.; Thevissen, K. Antifungal Plant Defensins: Mechanisms of Action and Production. Molecules 2014, 19 (8), 12280-12303. https://doi.org/10.3390/molecules190812280.

(14) Bulet, P.; Stöcklin, R. Insect Antimicrobial Peptides: Structures, Properties and Gene Regulation. Protein Pept. Lett. 2005, 12 (1), 3-11.

(15) Fehlbaum, P.; Bulet, P.; Michaut, L.; Lagueux, M.; Broekaert, W. F.; Hetru, C.; Hoffmann, J. A. Insect Immunity: Septic Injury of Drosophila Induces the Synthesis of a Potent Antifungal Peptide with Sequence Homology to Plant Antifungal Peptides. J. Biol. Chem. 1994, 269 (52), 33159-33163.

(16) Landon, C.; Sodano, P.; Hetru, C.; Hoffmann, J.; Ptak, M. Solution Structure of Drosomycin, the First Inducible Antifungal Protein from Insects. Protein Sci. 1997, 6 (9), 1878-1884. https://doi.org/10.1002/pro.5560060908.

(17) Lamberty, M.; Ades, S.; Uttenweiler-Joseph, S.; Brookhart, G.; Bushey, D.; Hoffmann, J. A.; Bulet, P. Insect Immunity: Isolation from the Lepidopteran Heliothis Virescens of a Novel Insect Defensin with Potent Antifungal Activity. J. Biol. Chem. 1999, 274 (14), 9320-9326. https://doi.org/10.1074/jbc.274.14.9320.

(18) Lamberty, M.; Caille, A.; Landon, C.; Tassin-Moindrot, S.; Hetru, C.; Bulet, P.; Vovelle, F. Solution Structures of the Antifungal Heliomicin and a Selected Variant with Both 
Antibacterial and Antifungal Activities. Biochemistry 2001, 40 (40), 11995-12003. https://doi.org/10.1021/bi0103563.

(19) Lamberty, M.; Zachary, D.; Lanot, R.; Bordereau, C.; Robert, A.; Hoffmann, J. A.; Bulet, P. Insect Immunity. Constitutive Expression of a Cysteine-Rich Antifungal and a Linear Antibacterial Peptide in a Termite Insect. J. Biol. Chem. 2001, 276 (6), 4085-4092. https://doi.org/10.1074/jbc.M002998200.

(20) Da Silva, P.; Jouvensal, L.; Lamberty, M.; Bulet, P.; Caille, A.; Vovelle, F. Solution Structure of Termicin, an Antimicrobial Peptide from the Termite Pseudacanthotermes Spiniger. Protein Sci. 2003, 12 (3), 438-446. https://doi.org/10.1110/ps.0228303.

(21) Schuhmann, B.; Seitz, V.; Vilcinskas, A.; Podsiadlowski, L. Cloning and Expression of Gallerimycin, an Antifungal Peptide Expressed in Immune Response of Greater Wax Moth Larvae, Galleria Mellonella. Arch. Insect Biochem. Physiol. 2003, 53 (3), 125-133. https://doi.org/10.1002/arch.10091.

(22) Landon, C.; Barbault, F.; Legrain, M.; Menin, L.; Guenneugues, M.; Schott, V.; Vovelle, F.; Dimarcq, J.-L. Lead Optimization of Antifungal Peptides with 3D NMR Structures Analysis. Protein Sci. 2004, 13 (3), 703-713. https://doi.org/10.1110/ps.03404404.

(23) Banzet, N.; Latorse, M.-P.; Bulet, P.; François, E.; Derpierre, C.; Dubald, M. Expression of Insect Cystein-Rich Antifungal Peptides in Transgenic Tobacco Enhances Resistance to a Fungal Disease. Plant Sci. 2002, 162 (6), 995-1006. https://doi.org/10.1016/S01689452(02)00053-5.

(24) Thevissen, K.; Kristensen, H.-H.; Thomma, B. P. H. J.; Cammue, B. P. A.; François, I. E. J. A. Therapeutic Potential of Antifungal Plant and Insect Defensins. Drug Discov. Today 2007, 12 (21), 966-971. https://doi.org/10.1016/j.drudis.2007.07.016.

(25) Andrès, E. Cationic Antimicrobial Peptides in Clinical Development, with Special Focus on Thanatin and Heliomicin. Eur. J. Clin. Microbiol. Infect. Dis. 2012, 31 (6), 881-888. https://doi.org/10.1007/s10096-011-1430-8.

(26) Halpern, N. Entomed mise sur le système immunitaire des insectes pour soigner l'homme. Les Echos. December 11, 2001.

(27) Aerts, A. M.; François, I. E. J. A.; Cammue, B. P. A.; Thevissen, K. The Mode of Antifungal Action of Plant, Insect and Human Defensins. Cell. Mol. Life Sci. 2008, 65 (13), 2069-2079. https://doi.org/10.1007/s00018-008-8035-0.

(28) Thevissen, K.; Warnecke, D. C.; François, I. E. J. A.; Leipelt, M.; Heinz, E.; Ott, C.; Zähringer, U.; Thomma, B. P. H. J.; Ferket, K. K. A.; Cammue, B. P. A. Defensins from Insects and Plants Interact with Fungal Glucosylceramides. J. Biol. Chem. 2004, 279 (6), 3900-3905. https://doi.org/10.1074/jbc.M311165200.

(29) Landon, C.; Vovelle, F.; Sodano, P.; Pajon, A. The Active Site of Drosomycin, a Small Insect Antifungal Protein, Delineated by Comparison with the Modeled Structure of RsAFP2, a Plant Antifungal Protein. J. Pept. Res. 2000, 56 (4), 231-238. https://doi.org/10.1034/j.1399-3011.2000.00757.x.

(30) Gao, B.; Zhu, S.-Y. Differential Potency of Drosomycin to Neurospora Crassa and Its Mutant: Implications for Evolutionary Relationship between Defensins from Insects and Plants. Insect Mol. Biol. 2008, 17 (4), 405-411. https://doi.org/10.1111/j.13652583.2008.00810.x.

(31) Rautenbach, M.; Troskie, A. M.; Vosloo, J. A. Antifungal Peptides: To Be or Not to Be Membrane Active. Biochimie 2016, 130, 132-145. https://doi.org/10.1016/j.biochi.2016.05.013. 
(32) Cools, T. L.; Struyfs, C.; Cammue, B. P.; Thevissen, K. Antifungal Plant Defensins: Increased Insight in Their Mode of Action as a Basis for Their Use to Combat Fungal Infections. Future Microbiol. 2017, 12 (5), 441-454. https://doi.org/10.2217/fmb-20160181 .

(33) El-Mounadi, K.; Islam, K. T.; Hernández-Ortiz, P.; Read, N. D.; Shah, D. M. Antifungal Mechanisms of a Plant Defensin MtDef4 Are Not Conserved between the Ascomycete Fungi Neurospora Crassa and Fusarium Graminearum. Mol. Microbiol. 2016, 100 (3), 542-559. https://doi.org/10.1111/mmi.13333.

(34) Parisi, K.; Shafee, T. M. A.; Quimbar, P.; van der Weerden, N. L.; Bleackley, M. R.; Anderson, M. A. The Evolution, Function and Mechanisms of Action for Plant Defensins. Semin. Cell Dev. Biol. 2018. https://doi.org/10.1016/j.semcdb.2018.02.004.

(35) Amselem, J.; Cuomo, C. A.; van Kan, J. A. L.; Viaud, M.; Benito, E. P.; Couloux, A.; Coutinho, P. M.; Vries, R. P. de; Dyer, P. S.; Fillinger, S.; et al. Genomic Analysis of the Necrotrophic Fungal Pathogens Sclerotinia Sclerotiorum and Botrytis Cinerea. PLOS Genet. 2011, 7 (8), e1002230. https://doi.org/10.1371/journal.pgen.1002230.

(36) González-Fernández, R.; Aloria, K.; Valero-Galván, J.; Redondo, I.; Arizmendi, J. M.; Jorrín-Novo, J. V. Proteomic Analysis of Mycelium and Secretome of Different Botrytis Cinerea Wild-Type Strains. J. Proteomics 2014, 97, 195-221. https://doi.org/10.1016/j.jprot.2013.06.022.

(37) van Kan, J. A. L.; Stassen, J. H. M.; Mosbach, A.; Lee, T. A. J. V. D.; Faino, L.; Farmer, A. D.; Papasotiriou, D. G.; Zhou, S.; Seidl, M. F.; Cottam, E.; et al. A Gapless Genome Sequence of the Fungus Botrytis Cinerea. Mol. Plant Pathol. 2017, 18 (1), 75-89. https://doi.org/10.1111/mpp.12384.

(38) Staats, M.; van Kan, J. A. L. Genome Update of Botrytis Cinerea Strains B05.10 and T4. Eukaryot. Cell 2012, 11 (11), 1413-1414. https://doi.org/10.1128/EC.00164-12.

(39) Liñeiro, E.; Cantoral, J. M.; Fernández-Acero, F. J. Contribution of Proteomics Research to Understanding Botrytis Biology and Pathogenicity. In Botrytis - the Fungus, the Pathogen and its Management in Agricultural Systems; 2016; pp 315-333.

(40) Fernández-Acero, F. J.; Jorge, I.; Calvo, E.; Vallejo, I.; Carbú, M.; Camafeita, E.; Garrido, C.; López, J. A.; Jorrin, J.; Cantoral, J. M. Proteomic Analysis of Phytopathogenic Fungus Botrytis Cinerea as a Potential Tool for Identifying Pathogenicity Factors, Therapeutic Targets and for Basic Research. Arch. Microbiol. 2007, 187 (3), 207-215. https://doi.org/10.1007/s00203-006-0188-3.

(41) González-Fernández, R.; Valero-Galván, J.; Gómez-Gálvez, F. J.; Jorrín-Novo, J. V. Unraveling the in Vitro Secretome of the Phytopathogen Botrytis Cinerea to Understand the Interaction with Its Hosts. Front. Plant Sci. 2015, 6. https://doi.org/10.3389/fpls.2015.00839.

(42) Shah, P.; Atwood, J. A.; Orlando, R.; El Mubarek, H.; Podila, G. K.; Davis, M. R. Comparative Proteomic Analysis of Botrytis Cinerea Secretome. J. Proteome Res. 2009, 8 (3), 1123-1130. https://doi.org/10.1021/pr8003002.

(43) Hou, Y.; Zheng, Z.; Xu, S.; Chen, C.; Zhou, M. Proteomic Analysis of Fusarium Graminearum Treated by the Fungicide JS399-19. Pestic. Biochem. Physiol. 2013, 107 (1), 86-92. https://doi.org/10.1016/j.pestbp.2013.05.009.

(44) Tanaka, S. Nutrition of Piricularia Oryzae in Vitro. In The rice blast disease; IRRI-Johns Hopkins Press: Baltimore, MD, 1965; pp 23-34. 
982

983

984

985

986

987

988

989

990

991

992

993

994

995

996

997

998

999

1000

1001

1002

1003

1004

1005

1006

1007

1008

1009

1010

1011

1012

1013

1014

1015

1016

1017

1018

1019

1020

1021

1022

1023

1024

1025

1026

1027

(45) Ou, S. H. Rice Diseases, 2nd ed.; Commonwealth Mycological Institute: Kew, Surrey, UK, 1985.

(46) Strohalm, M.; Kavan, D.; Novák, P.; Volný, M.; Havlíček, V. MMass 3: A Cross-Platform Software Environment for Precise Analysis of Mass Spectrometric Data. Anal. Chem. 2010, 82 (11), 4648-4651. https://doi.org/10.1021/ac100818g.

(47) Masson, V.; Arafah, K.; Voisin, S.; Bulet, P. Comparative Proteomics Studies of Insect Cuticle by Tandem Mass Spectrometry: Application of a Novel Proteomics Approach to the Pea Aphid Cuticular Proteins. PROTEOMICS 2018, 18 (3-4), 1700368. https://doi.org/10.1002/pmic.201700368.

(48) Pisani, C.; Voisin, S.; Arafah, K.; Durand, P.; Perrard, M.-H.; Guichaoua, M.-R.; Bulet, P.; Prat, O. Ex Vivo Assessment of Testicular Toxicity Induced by Carbendazim and Iprodione, Alone or in a Mixture. ALTEX - Altern. Anim. Exp. 2016, 33 (4), 393-413. https://doi.org/10.14573/altex.1601253.

(49) Eng, J. K.; McCormack, A. L.; Yates, J. R. An Approach to Correlate Tandem Mass Spectral Data of Peptides with Amino Acid Sequences in a Protein Database. J. Am. Soc. Mass Spectrom. 1994, 5 (11), 976-989. https://doi.org/10.1016/1044-0305(94)80016-2.

(50) Kersey, P. J.; Allen, J. E.; Allot, A.; Barba, M.; Boddu, S.; Bolt, B. J.; Carvalho-Silva, D.; Christensen, M.; Davis, P.; Grabmueller, C.; et al. Ensembl Genomes 2018: An Integrated Omics Infrastructure for Non-Vertebrate Species. Nucleic Acids Res. 2018, 46 (D1), D802-D808. https://doi.org/10.1093/nar/gkx1011.

(51) Käll, L.; Canterbury, J. D.; Weston, J.; Noble, W. S.; MacCoss, M. J. Semi-Supervised Learning for Peptide Identification from Shotgun Proteomics Datasets. Nat. Methods 2007, 4 (11), 923-925. https://doi.org/10.1038/nmeth1113.

(52) Käll, L.; Storey, J. D.; Noble, W. S. Qvality: Non-Parametric Estimation of q-Values and Posterior Error Probabilities. Bioinformatics 2009, 25 (7), 964-966. https://doi.org/10.1093/bioinformatics/btp021.

(53) Estabrook, R. W. Studies of Oxidative Phosphorylation with Potassium Ferricyanide as Electron Acceptor. J. Biol. Chem. 1961, 236 (11), 3051-3057.

(54) Terras, F. R.; Schoofs, H. M.; Bolle, M. F. D.; Leuven, F. V.; Rees, S. B.; Vanderleyden, J.; Cammue, B. P.; Broekaert, W. F. Analysis of Two Novel Classes of Plant Antifungal Proteins from Radish (Raphanus Sativus L.) Seeds. J. Biol. Chem. 1992, 267 (22), 1530115309.

(55) Nanni, V.; Schumacher, J.; Giacomelli, L.; Brazzale, D.; Sbolci, L.; Moser, C.; Tudzynski, P.; Baraldi, E. VvAMP2, a Grapevine Flower-Specific Defensin Capable of Inhibiting Botrytis Cinerea Growth: Insights into Its Mode of Action. Plant Pathol. 2014, 63 (4), 899-910. https://doi.org/10.1111/ppa.12170.

(56) Thevissen, K.; Terras, F. R. G.; Broekaert, W. F. Permeabilization of Fungal Membranes by Plant Defensins Inhibits Fungal Growth. Appl. Environ. Microbiol. 1999, 65 (12), $5451-5458$.

(57) Osborn, R. W.; Samblanx, G. W. D.; Thevissen, K.; Goderis, I.; Torrekens, S.; Leuven, F. V.; Attenborough, S.; Rees, S. B.; Broekaert, W. F. Isolation and Characterisation of Plant Defensins from Seeds of Asteraceae, Fabaceae, Hippocastanaceae and Saxifragaceae. FEBS Lett. 1995, 368 (2), 257-262. https://doi.org/10.1016/0014-5793(95)00666-W.

(58) Broekaert, W. F.; Terras, F.; Cammue, B.; Osborn, R. W. Plant Defensins: Novel Antimicrobial Peptides as Components of the Host Defense System. Plant Physiol. 1995, 108 (4), 1353-1358. https://doi.org/10.1104/pp.108.4.1353. 
1028

1029

1030

1031

1032

1033

1034

1035

1036

1037

1038

1039

1040

1041

1042

1043

1044

1045

1046

1047

(59) Spelbrink, R. G.; Dilmac, N.; Allen, A.; Smith, T. J.; Shah, D. M.; Hockerman, G. H. Differential Antifungal and Calcium Channel-Blocking Activity among Structurally Related Plant Defensins. Plant Physiol. 2004, 135 (4), 2055-2067. https://doi.org/10.1104/pp.104.040873.

(60) Schulthess, B.; Ledermann, R.; Mouttet, F.; Zbinden, A.; Bloemberg, G. V.; Böttger, E. C.; Hombach, M. Use of the Bruker MALDI Biotyper for Identification of Molds in the Clinical Mycology Laboratory. J. Clin. Microbiol. 2014, 52 (8), 2797-2803. https://doi.org/10.1128/JCM.00049-14.

(61) Thevissen, K.; Tavares, P. de M.; Xu, D.; Blankenship, J.; Vandenbosch, D.; IdkowiakBaldys, J.; Govaert, G.; Bink, A.; Rozental, S.; Groot, P. W. J. de; et al. The Plant Defensin RsAFP2 Induces Cell Wall Stress, Septin Mislocalization and Accumulation of Ceramides in Candida Albicans. Mol. Microbiol. 2012, 84 (1), 166-180. https://doi.org/10.1111/j.1365-2958.2012.08017.x.

(62) Funato, K.; Riezman, H. Vesicular and Nonvesicular Transport of Ceramide from ER to the Golgi Apparatus in Yeast. J. Cell Biol. 2001, 155 (6), 949-960. https://doi.org/10.1083/jcb.200105033.

(63) Hayes, B. M. E.; Bleackley, M. R.; Anderson, M. A.; Van der Weerden, N. L. The Plant Defensin NaD1 Enters the Cytoplasm of Candida Albicans via Endocytosis. J. Fungi 2018, 4 (1), 20. https://doi.org/10.3390/jof4010020. 


\section{For Table of Contents Only}

1049

1050

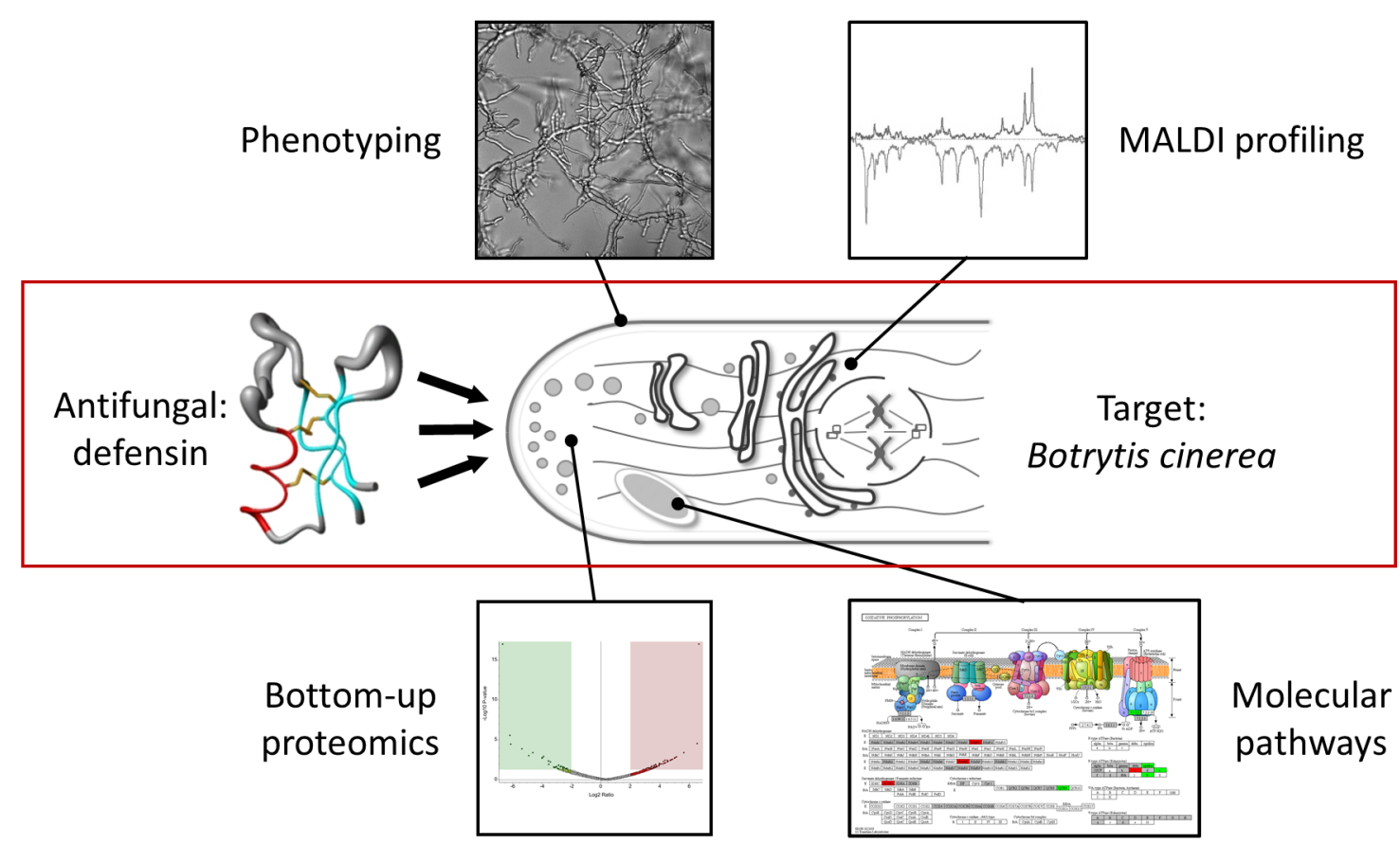

